Noname manuscript No.

(will be inserted by the editor)

\title{
Covariantized Noether identities and conservation laws for perturbations in metric theories of gravity
}

\author{
Alexander N. Petrov . \\ Robert R. Lompay
}

Received: date / Accepted: date

\begin{abstract}
A construction of conservation laws and conserved quantities for perturbations in arbitrary metric theories of gravity is developed. In an arbitrary field theory, with the use of incorporating an auxiliary metric into the initial Lagrangian covariantized Nœther identities are carried out. Identically conserved currents with corresponding superpotentials are united into a family. Such a generalized formalism of the covariantized identities gives a natural basis for constructing conserved quantities for perturbations. A new family of conserved currents and correspondent superpotentials for perturbations on arbitrary curved backgrounds in metric theories is suggested. The conserved quantities are both of pure canonical Nœther and of Belinfante corrected types. To test the results each of the superpotentials of the family is applied to calculate the mass of the Schwarzschild-anti-de Sitter black hole in the Einstein-Gauss-Bonnet gravity. Using all the superpotentials of the family gives the standard accepted mass.
\end{abstract}

Keywords Metric gravitation theories · differential identities · conservation laws

A. N. Petrov

Moscow MV Lomonosov State University, Sternberg Astronomical Institute, Universitetskii pr. 13, Moscow 119992, Russia

Tel.: $+7(495) 7315222$

Fax: +7(495)9328841

E-mail: alex.petrov55@gmail.com

R. R. Lompay

Department of Physics, Uzhgorod National University, Voloshyna str. 54, Uzhgorod 88000, Ukraine

Tel.: +380956940415

E-mail: rlompay@gmail.com 


\section{Introduction}

Examination and study of perturbations in general relativity (GR), including gravitational waves, cosmological perturbations, etc, unavoidably lead to constructing conservation laws and conserved quantities for such perturbations. To describe a perturbed model one needs to choose a background spacetime (not perturbed solution of the theory), in which perturbations propagate. Depending on a concrete problem, a background can be both flat (Minkowski spacetime) and arbitrary curved (e.g., cosmological, black hole, or another solution). Researches consider such systems both in an approximation (say, in linear one) and in an exact form (without any approximation, perturbations are not infinitesimal). In earlier works, conserved quantities in GR, classical pseudotensors and superpotentials, have been presented in a non-covariant form. At the present time, researches elaborate and study covariant quantities. For the most of the above one can recommend the reviews $[1,2]$ and numerous references therein; the paper [3] can be very useful also.

As a rule, a construction of conservation laws is based on using the Nother procedure. Even non-covariant pseudotensors and superpotentials have been connected with the Nœther theorem [4-6]. In the framework of GR, the Nœether procedure is applied to the Einstein-Hilbert action either directly, or not explicitly. Various appropriate modifications of the Lagrangian are used, these are an incorporation of background structures (such as metric, connection, etc), an addition of various divergences, etc (see, e.g., [7-13]). In GR, due to efforts of very many authors a technique of applying the Noether theorem has been well developed. However, last two decades, numerous metric theories, which are various modifications of GR, become more and more popular. They are quadratic in curvature theories, see, e.g., [14]; or theories of the Lovelock type [15]; or $f(R)$ theories [16], etc. For them there is also a necessity to study perturbations and construct conservation laws. Many results in this direction have been obtained also (see, for example, [17-23], and reviews [1, 2 ] and references therein). However, concrete forms of metric Lagrangians in such theories are very various and more complicated than in GR, therefore it is very desirable to elaborate united rules for constructing conservation laws for perturbations. The present paper is just devoted to this problem.

One of the main requirements is to construct covariant expressions and quantities. However, recall that covariant derivatives of the metric are equal to zero identically. Therefore a direct application of the Noether procedure in the framework of gravitational metric theories leads to non-covariant identities and conserved quantities [24]. To present covariant expressions one includes either additional structures, or uses relevant reformulations. One of the earlier attempts to suggest covariantized Nother identities in GR is the Ray work [25]. Here, in a definite sense, we develop Ray's ideas. Examining perturbed models with a fixed background (a known solution), we operate with a back-

ground metric anyway. Namely its presence permits us to construct covariant expressions. 
Already covariant identities and conserved quantities for perturbations in arbitrary field theories with using an auxiliary background metric have been suggested; these results have been applied in the framework of the EinsteinGauss-Bonnet (EGB) gravity [20-23]. However, first, a generalized formalism of constructing such conservation laws was not presented fully in all the details; second, not all the possibilities of such covariant generalized constructions have been discovered and developed. In the present paper, we close these gaps. The paper is organized as follows.

In Sect. 2, as preliminaries, we give Mitzkevich's presentation [24] for deriving Nother identities in an arbitrary field theory. The Noether procedure is applied directly to a covariant scalar density (Lagrangian), however presented in a not covariant form through partial derivatives of field variables up to a second order. All the metric theories, Lagrangians of which depend on the Riemann tensor algebraically, are just related to this class. As a finalized result, not covariant identically conserved currents and correspondent superpotentials are obtained.

In Sect. 3, covariant identities and identically conserved quantities are carried out in an arbitrary field theory. In Subsect. 3.1, for this goal one includes an arbitrary fixed external metric into the Lagrangian exchanging partial derivatives (in a special way) by covariant derivatives related to the external metric. In the result, the initial Lagrangian becomes evidently covariant, although in a reality it does not contain the auxiliary metric in whole. This trick permits us to present in a covariant form both identities and identically conserved quantities. It turns out that the way of constructing covariant conserved quantities presented in Subsect. 3.1 has a freedom, which is considered in Subsect. 3.2. In Subsect. 3.3, we explore the freedom to present a new family of covariant Nœther identities and identically conserved quantities. In Subsect. 3.4, we expand the new family using the Belinfante modification [26], which is a more popular reconstruction of the pure Nother procedure [13].

In Sect. 4, using the generalized results of Sect. 3, we consider an arbitrary metric theory. In Subsect. 4.1, necessary elements of the Nœether identities related to a pure metric gravitational Lagrangian are given. In Subsect. 4.2, a new family of covariant conservation laws and conserved quantities for perturbations on arbitrary curved backgrounds, containing both canonical Nœther and Belinfante corrected types, is presented.

In Sect. 5, we apply the above results to calculate a mass of D-dimensional black hole (BH) in the EGB gravity presented by the Schwartzchild-anti-de Sitter solution [27]. In Subsect. 5.1, we represent the new family of conservation laws for constructing conserved charges in EGB gravity in general. In Subsect. 5.2, we use them to calculate the black hole mass. Already, one of the variants of conservation laws included in the family has been used to study some solutions in EGB gravity [20-23]. We take into account the previous results as a start point for the present calculations.

In Sect. 6, we discuss the obtained results and future perspectives of their applications. In Appendix A, useful algebraic formulae for calculating with 
tensor densities in a generalized form are presented. In Appendix B, necessary formulae in the EGB gravity are given.

\section{Preliminaries. Nother identities in an arbitrary field theory}

In this section, our presentation follows the presentation in the book [24]. We derive the Nother identities and conserved quantities for arbitrary theories, an action of which

$$
S=\int d^{D} x \hat{L}
$$

is invariant with respect to coordinate transformations. Thus, the action (1) is a scalar, whereas the Lagrangian

$$
\hat{L}=\hat{L}\left(Q^{A} ; Q^{A}{ }_{, \alpha} ; Q^{A}{ }_{, \alpha \beta}\right)
$$

is a scalar density. Here and below 'hat' means that a quantity is a density of the wight +1 , for example, $\hat{g}^{\mu \nu}=\sqrt{-g} g^{\mu \nu}, \hat{L}=\sqrt{-g} L$. Here, the covariance of the action (or more formally, of the Lagrangian) is the basis for applying the Nother procedure. Dynamical fields of the system (1) and (2) are presented by a set of tensor densities $Q^{A}$, where the generalized index 'A' is a collective tensor index. Keeping in mind popular metric gravitational theories, the Lagrangian includes derivatives up to a second order. Here and below, Greek indexes numerate coordinates in $D$-dimensional spacetime; $(, \alpha) \equiv \partial_{\alpha}$ are partial (ordinary) derivatives.

Considering Lie displacements as perturbations of the system, we define variations of fields as their Lie derivatives:

$$
\delta_{\xi} Q^{A}=£_{\xi} Q^{A}=-\xi^{\alpha} \partial_{\alpha} Q^{A}+\left.Q^{A}\right|_{\beta} ^{\alpha} \partial_{\alpha} \xi^{\beta} .
$$

Please, note the opposite sign 'minus' with respect to the usual one (we follow the definition of variations in [24]). The notation $\left.Q^{A}\right|_{\beta} ^{\alpha}$ is defined by the transformation properties of $Q^{A}$; algebraic properties of $\left.Q^{A}\right|_{\beta} ^{\alpha}$, necessary here, can be found in Appendix A.

Because the Lagrangian is a scalar density of the weight +1 , its variation leads to the identity:

$$
\delta_{\xi} \hat{L}=£_{\xi} \hat{L} \equiv-\left(\xi^{\alpha} \hat{L}\right)_{, \alpha} .
$$

Keeping in mind a dependence of the Lagrangian (2) on $Q^{A}$ and their derivatives and substituting (3) into (4) one obtains the identity:

$$
\frac{\delta \hat{L}}{\delta Q_{B}} £_{\xi} Q_{B}+\partial_{\alpha}\left[\frac{\delta \hat{L}}{\delta Q_{B, \alpha}} £_{\xi} Q_{B}+\frac{\partial \hat{L}}{\partial Q_{B, \beta \alpha}}\left(£_{\xi} Q_{B}\right)_{, \beta}+\xi^{\alpha} \hat{L}\right] \equiv 0 .
$$

Usually the identity (5) is named as the general Nœther identity. The Lagrangian derivative is defined as usual

$$
\frac{\delta \hat{L}}{\delta Q_{B}}=\frac{\partial \hat{L}}{\partial Q_{B}}-\partial_{\alpha}\left(\frac{\partial \hat{L}}{\partial Q_{B, \alpha}}\right)+\partial_{\alpha \beta}\left(\frac{\partial \hat{L}}{\partial Q_{B, \alpha \beta}}\right)
$$


(where $\partial_{\beta \tau} \equiv \partial_{\beta} \partial_{\tau}$ ) and defines the left hand side of the field equations for $Q_{B}$ :

$$
\frac{\delta \hat{L}}{\delta Q_{B}}=0
$$

We introduce also the notation:

$$
\frac{\delta \hat{L}}{\delta Q_{B, \alpha}} \equiv \frac{\partial \hat{L}}{\partial Q_{B, \alpha}}-\partial_{\beta}\left(\frac{\partial \hat{L}}{\partial Q_{B, \alpha \beta}}\right) .
$$

Substituting (3) into (5) and providing identical transformations one obtains

$$
\begin{aligned}
& -\left[\frac{\delta \hat{L}}{\delta Q_{B}} Q_{B, \alpha}+\partial_{\beta}\left(\left.\frac{\delta \hat{L}}{\delta Q_{B}} Q_{B}\right|_{\alpha} ^{\beta}\right)\right] \xi^{\alpha} \\
& +\partial_{\alpha}\left[\hat{U}_{\sigma}{ }^{\alpha} \xi^{\sigma}+\hat{M}_{\sigma}{ }^{\alpha \tau} \partial_{\tau} \xi^{\sigma}+\hat{N}_{\sigma}{ }^{\alpha \tau \beta} \partial_{\beta \tau} \xi^{\sigma}\right] \equiv 0 .
\end{aligned}
$$

In (9), the coefficients are defined by the Lagrangian without ambiguities in unique way:

$$
\begin{gathered}
\hat{U}_{\sigma}{ }^{\alpha} \equiv \hat{L} \delta_{\sigma}^{\alpha}+\left.\frac{\delta \hat{L}}{\delta Q_{B}} Q_{B}\right|_{\sigma} ^{\alpha}-\frac{\delta \hat{L}}{\delta Q_{B, \alpha}} \partial_{\sigma} Q_{B}-\frac{\partial \hat{L}}{\partial Q_{B, \beta \alpha}} \partial_{\beta \sigma} Q_{B}, \\
\left.\hat{M}_{\sigma}{ }^{\alpha \tau} \equiv \frac{\delta \hat{L}}{\delta Q_{B, \alpha}} Q_{B}\right|_{\sigma} ^{\tau}-\frac{\partial \hat{L}}{\partial Q_{B, \tau \alpha}} \partial_{\sigma} Q_{B}+\frac{\partial \hat{L}}{\partial Q_{B, \beta \alpha}} \partial_{\beta}\left(\left.Q_{B}\right|_{\sigma} ^{\tau}\right), \\
\hat{N}_{\sigma}{ }^{\alpha \tau \beta} \equiv \frac{1}{2}\left[\left.\frac{\partial \hat{L}}{\partial Q_{B, \beta \alpha}} Q_{B}\right|_{\sigma} ^{\tau}+\left.\frac{\partial \hat{L}}{\partial Q_{B, \tau \alpha}} Q_{B}\right|_{\sigma} ^{\beta}\right] .
\end{gathered}
$$

To derive the last coefficient the evident relation $\hat{N}_{\sigma}{ }^{\alpha \tau \beta}=\hat{N}_{\sigma}{ }^{\alpha \beta \tau}$, following from (9), has been used.

Opening the identity (9) and, since $\xi^{\sigma}, \partial_{\alpha} \xi^{\sigma}, \partial_{\beta \alpha} \xi^{\sigma}$ and $\partial_{\gamma \beta \alpha} \xi^{\sigma}$ are arbitrary at every world point, we equalize to zero the coefficients at them independently and obtain the system of identities:

$$
\begin{aligned}
& \partial_{\alpha} \hat{U}_{\sigma}{ }^{\alpha} \equiv \frac{\delta \hat{L}}{\delta Q_{B}} Q_{B, \alpha}+\partial_{\beta}\left(\left.\frac{\delta \hat{L}}{\delta Q_{B}} Q_{B}\right|_{\alpha} ^{\beta}\right), \\
& \hat{U}_{\sigma}^{\alpha}+\partial_{\lambda} \hat{M}_{\sigma}^{\lambda \alpha} \equiv 0 \\
& \hat{M}_{\sigma}^{(\alpha \beta)}+\partial_{\lambda} \hat{N}_{\sigma}^{\lambda(\alpha \beta)} \equiv 0 \\
& \hat{N}_{\sigma}^{(\alpha \beta \gamma)} \equiv 0
\end{aligned}
$$

As we know, the system of equations (13) - (16) was pioneered by Klein [28, 29]. Therefore, we shall refer to this system as the Klein identities. After differentiating (14) and using (15) and (16) one obtains the identity $\partial_{\alpha} \hat{U}_{\sigma}{ }^{\alpha} \equiv 0$. This means that the right hand side of (13) is equal identically to zero also

$$
\frac{\delta \hat{L}}{\delta Q_{B}} Q_{B, \alpha}+\partial_{\beta}\left(\left.\frac{\delta \hat{L}}{\delta Q_{B}} Q_{B}\right|_{\alpha} ^{\beta}\right) \equiv 0 .
$$


These are just the Nœther identities. Thus instead of the identity (9) one can use independently (17) and

$$
\partial_{\alpha}\left[\hat{U}_{\sigma}^{\alpha} \xi^{\sigma}+\hat{M}_{\sigma}^{\alpha \tau} \partial_{\tau} \xi^{\sigma}+\hat{N}_{\sigma}{ }^{\alpha \tau \beta} \partial_{\beta \tau} \xi^{\sigma}\right] \equiv 0 .
$$

Call the expression under the divergence with a sign 'minus' as a current

$$
\hat{I}^{\alpha}(\xi)=-\left[\hat{U}_{\sigma}{ }^{\alpha} \xi^{\sigma}+\hat{M}_{\sigma}{ }^{\alpha \tau} \partial_{\tau} \xi^{\sigma}+\hat{N}_{\sigma}{ }^{\alpha \tau \beta} \partial_{\beta \tau} \xi^{\sigma}\right] .
$$

Sign 'minus' is selected to be in a correspondence with the usual sign 'minus' before gravitational (metric) action (see, e.g., [1]). Because the divergence (18) is equal to zero identically, the current has to be expressed through a quantity (superpotential), a double divergence of which has to be equal to zero identically: $\hat{I}^{\alpha}(\xi) \equiv \partial_{\beta} \hat{I}^{\alpha \beta}(\xi)$, where $\partial_{\alpha \beta} \hat{I}^{\alpha \beta}(\xi) \equiv 0$. Let us show this. Due to the symmetry in last two indexes in (12) and the identity (16) one has

$$
\hat{N}_{\sigma}^{\alpha \tau \beta}+\hat{N}_{\sigma}^{\tau \beta \alpha}+\hat{N}_{\sigma}^{\beta \alpha \tau} \equiv 0 .
$$

Using this identity and (14), after not complicated transformations one obtains

$$
\begin{aligned}
\hat{I}^{\alpha}(\xi) & =\partial_{\beta}\left(\hat{M}_{\sigma}^{\beta \alpha} \xi^{\sigma}+2 \hat{N}_{\sigma}^{\beta \alpha \lambda} \xi_{, \lambda}^{\sigma}\right) \\
& =\partial_{\beta}\left[\hat{M}_{\sigma}^{[\beta \alpha]} \xi^{\sigma}+2 \hat{N}_{\sigma}^{[\beta \alpha] \lambda} \xi_{, \lambda}^{\sigma}-\partial_{\lambda}\left(\hat{N}_{\sigma}^{\lambda \alpha \beta} \xi^{\sigma}\right)\right] .
\end{aligned}
$$

This is rewritten in the form of the identity

$$
\hat{I}^{\alpha}(\xi) \equiv \partial_{\beta} \hat{I}^{\alpha \beta}
$$

where the superpotential is defined as

$$
\hat{I}^{\alpha \beta}(\xi)=-\left[\hat{M}_{\sigma}^{[\alpha \beta]} \xi^{\sigma}+2 \hat{N}_{\sigma}^{[\alpha \beta] \lambda} \xi_{, \lambda}^{\sigma}+\partial_{\lambda}\left(\hat{N}_{\sigma}^{\lambda \alpha \beta} \xi^{\sigma}\right)\right] .
$$

It is evident that $\partial_{\alpha \beta} \hat{I}^{\alpha \beta}(\xi) \equiv 0$, the last term disappears under the double divergence due to the identity (16). Thus (22) can be considered as the identity equivalent to the conservation law (18) for the current $\hat{I}^{\alpha}(\xi)$.

It is not a goal of the present paper to describe and discuss nuances of the Nœther theorems [30] in a detail. For this important and interesting topic one can see, e.g., [31-35] and references there in. Here, the above presentation differs by the form from the results of applying the classically formulated Nœther theorems. It is because the presentation (1) - (23) is more convenient for deriving conserved quantities in metric theories of gravity. Although, of course, an analysis of the general Nœther identity has to cover the classical presentation. Let us show this.

The first Nother theorem can be formulated as follows: If the action $S$ is invariant under a finite continuous group of transformations $G_{r}$ depending smoothly on $r$ independent parameters, then there exist $r$ linearly independent combinations of the operators of the field equations (Lagrangian derivatives 
(6)) expressed through divergences of currents. Let us substitute $\xi^{\alpha}=\varepsilon^{\alpha}=$ const into (5). Then one obtains the identity:

$$
\frac{\delta \hat{L}}{\delta Q_{B}} Q_{B, \alpha} \equiv \partial_{\beta}\left(\delta_{\alpha}^{\beta} \hat{L}-\frac{\delta \hat{L}}{\delta Q_{B, \beta}} Q_{B, \alpha}-\frac{\partial \hat{L}}{\partial Q_{B, \gamma \beta}} Q_{B, \alpha \gamma}\right) \equiv \partial_{\beta} \hat{J}_{(\alpha)}^{\beta},
$$

which is just the result of applying the first Nœether theorem. Here, the currents are defined as $\hat{J}_{(\alpha)}^{\beta}$ and are conserved, $\partial_{\beta} \hat{J}_{(\alpha)}^{\beta}=0$, if the field equations are satisfied: $\delta \hat{L} / \delta Q_{B}=0$.

The second Nœether theorem can be formulated as follows: If the action $S$ is invariant under an infinite continuous group of transformations $G_{\infty r}$ depending smoothly on $r$ arbitrary functions, then there exist $r$ identically vanishing linearly independent combinations of Lagrangian derivatives and their partial derivatives. The identity (17) is the result of applying this theorem.

Combining the results of the first and second theorems, Nœether has formulated the statement often referred as the third Nœther theorem [31-35], although it was not presented as a theorem. It is explicitly presented in section 6: "An Assertion of Hilbert" of her paper [36,30] and sounds as follows: If the action $S$ is invariant under an infinite continuous group of transformations $G_{\infty r}$, then the current $\hat{J}_{(\alpha)}^{\beta}$ constructed for a finite subgroup $G_{r}$ of the group $G_{\infty r}$ (due to the first Nother theorem) is the sum of the divergence of the superpotential and a term vanishing on the equations of motion. However, Nœther did not provide a recipe for the superpotential construction. In the case of generally covariant theories, an analogous statement was proven by Klein (the Klein boundary theorem) in his works $[28,29]$ that appeared prior to the Nœther's paper where they are cited. Furthermore, additionally to the identities (14) - (16), Klein supplied the recipe for the superpotential construction. Taking into account such a historical development, we shall name the system (14) - (16) and (17) as the Klein-Nother identities.

All the above expressions and identities are not covariant. The next sections are devoted to a construction of covariant identities and conserved quantities.

\section{Covariantization of Nœether identities by including external (background) metric}

\subsection{A direct application of the Nœther procedure}

The Lagrangian (2) of a covariant theory is not covariant evidently. One of the ways to present it in an explicitly covariant form is to incorporate an external (auxiliary, background) metric as follows. Turning to the formula (163) one finds

$$
\begin{aligned}
Q_{B ; \alpha} & \equiv Q_{B, \alpha}+\left.\bar{\Gamma}_{\alpha \rho}^{\tau} Q_{B}\right|_{\tau} ^{\rho}, \\
Q_{B ; \alpha \beta} & \equiv Q_{B, \alpha \beta}+\left.\bar{\Gamma}_{\alpha \rho, \beta}^{\tau} Q_{B}\right|_{\tau} ^{\rho}+\bar{\Gamma}_{\alpha \rho}^{\tau}\left(\left.Q_{B}\right|_{\tau} ^{\rho}\right)_{, \beta}
\end{aligned}
$$




$$
\begin{aligned}
& +\bar{\Gamma}_{\beta \rho}^{\tau}\left[\left(\left.Q_{B}\right|_{\tau} ^{\rho}\right)_{, \alpha}+\left.\left.\bar{\Gamma}_{\alpha \nu}^{\mu} Q_{B}\right|_{\tau} ^{\rho}\right|_{\mu} ^{\nu}-\delta_{\alpha}^{\rho}\left(Q_{B, \tau}+\left.\bar{\Gamma}_{\tau \nu}^{\mu} Q_{B}\right|_{\mu} ^{\nu}\right)\right] \\
& \equiv Q_{B, \alpha \beta}+\left.\bar{\Gamma}_{\alpha \rho, \beta}^{\tau} Q_{B}\right|_{\tau} ^{\rho}+\bar{\Gamma}_{\alpha \rho}^{\tau}\left(\left.Q_{B}\right|_{\tau} ^{\rho}\right)_{; \beta}+\left.\bar{\Gamma}_{\beta \rho}^{\tau}\left(Q_{B ; \alpha}\right)\right|_{\tau} ^{\rho} \\
& -\left.\left.\bar{\Gamma}_{\alpha \rho}^{\tau} \bar{\Gamma}_{\beta \nu}^{\mu} Q_{B}\right|_{\tau} ^{\rho}\right|_{\mu} ^{\nu} .
\end{aligned}
$$

Because the Lagrangian (2) is a scalar density, after identical substitutions

$$
\begin{aligned}
Q_{B, \alpha} & \equiv Q_{B ; \alpha}-\left.\bar{\Gamma}_{\alpha \rho}^{\tau} Q_{B}\right|_{\tau} ^{\rho}, \\
Q_{B, \alpha \beta} & \equiv Q_{B ; \alpha \beta}-\left.\bar{\Gamma}_{\alpha \rho, \beta}^{\tau} Q_{B}\right|_{\tau} ^{\rho}-\bar{\Gamma}_{\alpha \rho}^{\tau}\left(\left.Q_{B}\right|_{\tau} ^{\rho}\right)_{; \beta}-\left.\bar{\Gamma}_{\beta \rho}^{\tau}\left(Q_{B ; \alpha}\right)\right|_{\tau} ^{\rho} \\
& +\left.\left.\bar{\Gamma}_{\alpha \rho}^{\tau} \bar{\Gamma}_{\beta \nu}^{\mu} Q_{B}\right|_{\tau} ^{\rho}\right|_{\mu} ^{\nu},
\end{aligned}
$$

it is transformed into an explicitly covariant form:

$$
\hat{L}\left(Q_{B}, Q_{B, \alpha}, Q_{B, \alpha \beta}\right) \equiv \hat{\mathcal{L}}\left(Q_{B}, Q_{B ; \alpha}, Q_{B ; \alpha \beta}, \bar{g}_{\mu \nu}, \bar{R}_{\mu \beta \nu}^{\alpha}\right) .
$$

Here, $\bar{g}_{\mu \nu}, \bar{\Gamma}_{\alpha \nu}^{\mu}$ and $\bar{R}_{\mu \beta \nu}^{\alpha}$ are metric, Cristoffel symbols and the curvature tensor of the auxiliary spacetime; $\left({ }_{; \alpha}\right)=\bar{D}_{\alpha}$ means a covariant derivative with respect to $\bar{g}_{\mu \nu}$; here and below 'bar' means that a quantity is a background one. One needs to make an important remark. The left hand side of (28) is evidently symmetric in $\alpha$ and $\beta$. To show this for the right hand side one has to present $Q_{B ; \alpha \beta}=Q_{B ;(\alpha \beta)}+Q_{B ;[\alpha \beta]}$, turn to the formula (167) and make necessary algebraic transformations using other formulae from Appendix A.

To conserve the explicit covariance under variation of $\hat{\mathcal{L}}$ the direct way is to variate the external metric $\bar{g}_{\mu \nu}$ together with fields $Q_{B}$. However, this way is very cumbersome, and we are going by a more economical one. It is easily to check that the right hand sides of (27) and (28) do not contain the background metric and Christoffel symbols in a reality. To be convinced in this one has to open covariant derivatives in the explicit form. Then it is clear that substitution of (27) and (28) does not incorporate an additional external metric. Recall that the initial Lagrangian (the left hand side of (29)) does not contain a background metric by definition. Therefore the new presentation of the initial Lagrangian after substitution of (27) and (28), namely $\hat{\mathcal{L}}$ in (29), does not contain $\bar{g}_{\mu \nu}$ and its derivatives in whole. This means that finally variation of $\hat{\mathcal{L}}$ has to be transformed into the identity (9) anyway. Thus, we follow the inverse way. Using (29), we represent (9) into an explicitly covariant form, and then obtain covariant identities and covariant conserved quantities.

At first we note that the identity (9) is covariant in whole since it has been obtained from the covariant identity (4) directly and conserving all the terms. Now, turn to the identity (17). It is known that the Lagrangian derivative of the scalar density (6) is covariant. Here, it is useful to demonstrate this fact and to have a covariant expression at hand. We use the algebraic properties of the quantities $\left.Q_{B}\right|_{\tau} ^{\rho}$ given in Appendix A. Let us consider the terms of (6) separately, the first one can be represented as

$$
\frac{\partial \hat{L}}{\partial Q_{B}}=\frac{\partial \hat{\mathcal{L}}}{\partial Q_{B}}+\frac{\partial \hat{\mathcal{L}}}{\partial Q_{C ; \alpha}} \frac{\partial Q_{C ; \alpha}}{\partial Q_{B}}+\frac{\partial \hat{\mathcal{L}}}{\partial Q_{C ; \alpha \beta}} \frac{\partial Q_{C ; \alpha \beta}}{\partial Q_{B}}
$$




$$
\begin{aligned}
& =\frac{\partial \hat{\mathcal{L}}}{\partial Q_{B}}+\frac{\partial \hat{\mathcal{L}}}{\partial Q_{C ; \alpha}} \frac{\partial}{\partial Q_{B}}\left(\left.\bar{\Gamma}_{\alpha \tau}^{\rho} Q_{C}\right|_{\rho} ^{\tau}\right)+\frac{\partial \hat{\mathcal{L}}}{\partial Q_{C ; \alpha \beta}} \frac{\partial}{\partial Q_{B}}\left[\left.\bar{\Gamma}_{\alpha \tau, \beta}^{\rho} Q_{C}\right|_{\rho} ^{\tau}\right. \\
& \left.+\left(\left.\left.\delta_{\alpha}^{\sigma} Q_{C}\right|_{\rho} ^{\tau}\right|_{\nu} ^{\mu}-\left.\delta_{\rho}^{\sigma} \delta_{\alpha}^{\tau} Q_{C}\right|_{\nu} ^{\mu}\right) \bar{\Gamma}_{\beta \tau}^{\rho} \bar{\Gamma}_{\mu \sigma}^{\nu}\right] .
\end{aligned}
$$

To derive the second term in (6) it is necessary the next equality, which follows from (26) and (29):

$$
\begin{aligned}
\frac{\partial \hat{L}}{\partial Q_{B, \alpha}} & =\frac{\partial \hat{\mathcal{L}}}{\partial Q_{B ; \alpha}}+\frac{\partial \hat{\mathcal{L}}}{\partial Q_{C ; \mu \nu}} \frac{\partial Q_{C ; \mu \nu}}{\partial Q_{B ; \alpha}} \\
& =\frac{\partial \hat{\mathcal{L}}}{\partial Q_{B ; \alpha}}+\frac{\partial \hat{\mathcal{L}}}{\partial Q_{C ; \mu \nu}} \frac{\partial}{\partial Q_{B ; \alpha}}\left[\bar{\Gamma}_{\mu \rho}^{\tau}\left(\left.Q_{C}\right|_{\tau} ^{\rho}\right)_{; \nu}+\left.\bar{\Gamma}_{\nu \rho}^{\tau}\left(Q_{C ; \mu}\right)\right|_{\tau} ^{\rho}\right]
\end{aligned}
$$

Thus for the second term in (6) one has

$$
\begin{aligned}
-\partial_{\alpha}\left(\frac{\partial \hat{L}}{\partial Q_{B, \alpha}}\right) & =-\bar{D}_{\alpha}\left(\frac{\partial \hat{\mathcal{L}}}{\partial Q_{B ; \alpha}}\right)+\left.\bar{\Gamma}_{\alpha \rho}^{\tau}\left(\frac{\partial \hat{\mathcal{L}}}{\partial Q_{B ; \alpha}}\right)\right|_{\tau} ^{\rho} \\
& -\partial_{\alpha}\left(\frac{\partial \hat{\mathcal{L}}}{\partial Q_{C ; \mu \nu}} \frac{\partial}{\partial Q_{B ; \alpha}}\left[\bar{\Gamma}_{\mu \rho}^{\tau}\left(\left.Q_{C}\right|_{\tau} ^{\rho}\right)_{; \nu}+\left.\bar{\Gamma}_{\nu \rho}^{\tau}\left(Q_{C ; \mu}\right)\right|_{\tau} ^{\rho}\right]\right) .(32)
\end{aligned}
$$

To derive the third term in (6) we note that

$$
\frac{\partial \hat{L}}{\partial Q_{B, \alpha \beta}}=\frac{\partial \hat{\mathcal{L}}}{\partial Q_{B ; \alpha \beta}}
$$

it is evidently covariant and directly follows from (26) and (29). (Here, we do not take into account the symmetry in $\alpha$ and $\beta$ at the left hand side, see discussion in Subsect. 3.2.) Thus for the third term in (6) one has

$$
\begin{aligned}
\partial_{\alpha \beta}\left(\frac{\partial \hat{L}}{\partial Q_{B, \alpha \beta}}\right) & =\bar{D}_{\alpha \beta}\left(\frac{\partial \hat{\mathcal{L}}}{\partial Q_{B ; \alpha \beta}}\right)-\left.\bar{\Gamma}_{\alpha \rho}^{\tau}\left[\bar{D}_{\beta} \frac{\partial \hat{\mathcal{L}}}{\partial Q_{B ; \alpha \beta}}\right]\right|_{\tau} ^{\rho} \\
& -\partial_{\alpha}\left[\left.\bar{\Gamma}_{\beta \rho}^{\tau}\left(\frac{\partial \hat{\mathcal{L}}}{\partial Q_{B ; \alpha \beta}}\right)\right|_{\tau} ^{\rho}\right]
\end{aligned}
$$

where $\bar{D}_{\alpha \beta}=\bar{D}_{\alpha} \bar{D}_{\beta}$. Summing equalities (30), (32) and (34) one finds that the first terms on the right hand sides survive, the other terms are selfcompensated due to the rules in Appendix A. Thus we show that the Lagrangian derivative (6), the left hand side of the equations of motion (7), is represented in the explicitly covariant form:

$$
\frac{\delta \hat{L}}{\delta Q_{B}}=\frac{\delta \hat{\mathcal{L}}}{\delta Q_{B}}=\frac{\partial \hat{\mathcal{L}}}{\partial Q_{B}}-\bar{D}_{\alpha}\left(\frac{\partial \hat{\mathcal{L}}}{\partial Q_{B ; \alpha}}\right)+\bar{D}_{\alpha \beta}\left(\frac{\partial \hat{\mathcal{L}}}{\partial Q_{B ; \alpha \beta}}\right) .
$$


Keeping this in mind and using the properties discussed in Appendix A, it is not difficult to show that (17) has also a covariant form

$$
\frac{\delta \hat{\mathcal{L}}}{\delta Q_{B}} Q_{B ; \alpha}+\bar{D}_{\beta}\left(\left.\frac{\delta \hat{\mathcal{L}}}{\delta Q_{B}} Q_{B}\right|_{\alpha} ^{\beta}\right) \equiv 0 .
$$

Then one concludes that the identity (18), the same as (9), is covariant in whole.

Now, let us change partial derivatives of $\xi^{\sigma}$ in (18) in the way $\partial_{\rho} \xi^{\sigma}=$ $\bar{D}_{\rho} \xi^{\sigma}-\left.\xi^{\sigma}\right|_{\beta} ^{\alpha} \bar{\Gamma}_{\rho \alpha}^{\beta}$ and rewrite it as

$$
\partial_{\alpha}\left[\hat{u}_{\sigma}{ }^{\alpha} \xi^{\sigma}+\hat{m}_{\sigma}{ }^{\alpha \tau} \bar{D}_{\tau} \xi^{\sigma}+\hat{n}_{\sigma}{ }^{\alpha \tau \beta} \bar{D}_{\beta \tau} \xi^{\sigma}\right] \equiv 0
$$

where

$$
\begin{aligned}
\hat{u}_{\sigma}{ }^{\alpha} & =\hat{U}_{\sigma}{ }^{\alpha}-\hat{M}_{\lambda}{ }^{\alpha \tau} \Gamma_{\sigma \tau}^{\lambda}+\hat{N}_{\lambda}{ }^{\alpha \tau \rho}\left(\Gamma_{\tau \pi}^{\lambda} \Gamma_{\sigma \rho}^{\pi}-\partial_{\rho} \Gamma_{\sigma \tau}^{\lambda}\right), \\
\hat{m}_{\sigma}{ }^{\alpha \tau} & =\hat{M}_{\sigma}{ }^{\alpha \tau}+\hat{N}_{\sigma}{ }^{\alpha \lambda \rho} \Gamma_{\lambda \rho}^{\tau}-2 \hat{N}_{\lambda}{ }^{\alpha \tau \rho} \Gamma_{\sigma \rho}^{\lambda}, \\
\hat{n}_{\sigma}{ }^{\alpha \tau \beta} & =\hat{N}_{\sigma}{ }^{\alpha \tau \beta} .
\end{aligned}
$$

Below, with using the new form of the Lagrangian (29) and the connections between partial and covariant derivatives (25) and (26) we show that the new coefficients (38) - (40) are represented in an explicitly covariant form.

Let us begin from the last the coefficient (40). Due to (33) the coefficient $N$ (12) is automatically covariant, and the coefficient $n$ is presented in the obviously covariant form

$$
\hat{n}_{\sigma}{ }^{\alpha \tau \beta} \equiv \frac{1}{2}\left[\left.\frac{\partial \hat{\mathcal{L}}}{\partial Q_{B ; \beta \alpha}} Q_{B}\right|_{\sigma} ^{\tau}+\left.\frac{\partial \hat{\mathcal{L}}}{\partial Q_{B ; \tau \alpha}} Q_{B}\right|_{\sigma} ^{\beta}\right] .
$$

To represent $m$ in (39) we need in the representation of $M$ in (11). The first term in $M$ is defined by the derivative (8), let us reproduce it. For this we use (31), (33), and rules in Appendix A. One gets

$$
\begin{aligned}
\frac{\delta \hat{L}}{\delta Q_{B, \alpha}} & =\frac{\partial \hat{\mathcal{L}}}{\partial Q_{B ; \alpha}}+\frac{\partial \hat{\mathcal{L}}}{\partial Q_{B ; \mu \nu}} \frac{\partial}{\partial Q_{B ; \alpha}}\left[\bar{\Gamma}_{\mu \rho}^{\tau}\left(\left.Q_{B}\right|_{\tau} ^{\rho}\right)_{; \nu}+\left.\bar{\Gamma}_{\nu \rho}^{\tau}\left(Q_{B ; \mu}\right)\right|_{\tau} ^{\rho}\right] \\
& -\bar{D}_{\beta}\left(\frac{\partial \hat{\mathcal{L}}}{\partial Q_{B ; \alpha \beta}}\right)+\left.\bar{\Gamma}_{\beta \mu}^{\nu}\left(\frac{\partial \hat{\mathcal{L}}}{\partial Q_{B ; \alpha \beta}}\right)\right|_{\nu} ^{\mu} .
\end{aligned}
$$

Using this expression and (33), and again rules in Appendix A, one obtains finally for $M$ in (11):

$$
\begin{aligned}
\hat{M}_{\sigma}^{\alpha \tau} & \equiv\left[\frac{\partial \hat{\mathcal{L}}}{\partial Q_{B ; \alpha}}+\frac{\partial \hat{\mathcal{L}}}{\partial Q_{B ; \mu \nu}} \frac{\partial}{\partial Q_{B ; \alpha}}\left[\bar{\Gamma}_{\mu \rho}^{\tau}\left(\left.Q_{B}\right|_{\tau} ^{\rho}\right)_{; \nu}+\left.\bar{\Gamma}_{\nu \rho}^{\tau}\left(Q_{B ; \mu}\right)\right|_{\tau} ^{\rho}\right]\right. \\
& \left.-\bar{D}_{\beta}\left(\frac{\partial \hat{\mathcal{L}}}{\partial Q_{B ; \alpha \beta}}\right)+\left.\bar{\Gamma}_{\beta \mu}^{\nu}\left(\frac{\partial \hat{\mathcal{L}}}{\partial Q_{B ; \alpha \beta}}\right)\right|_{\nu} ^{\mu}\right]\left.Q_{B}\right|_{\sigma} ^{\tau}
\end{aligned}
$$




$$
\begin{aligned}
& -\frac{\partial \hat{\mathcal{L}}}{\partial Q_{B ; \tau \alpha}}\left[\bar{D}_{\sigma} Q_{B}-\left.\bar{\Gamma}_{\sigma \mu}^{\nu} Q_{B}\right|_{\nu} ^{\mu}\right] \\
& +\frac{\partial \hat{\mathcal{L}}}{\partial Q_{B ; \beta \alpha}}\left[\bar{D}_{\beta}\left(\left.Q_{B}\right|_{\sigma} ^{\tau}\right)-\left.\left.\bar{\Gamma}_{\beta \mu}^{\nu} Q_{B}\right|_{\sigma} ^{\tau}\right|_{\nu} ^{\mu}\right] .
\end{aligned}
$$

Next, substituting (43), (40) and (41) into (39) and, after using the rules of Appendix A, one gets the evidently covariant form for $m$ :

$$
\begin{aligned}
\hat{m}_{\sigma}{ }^{\alpha \tau} & \left.\equiv\left[\frac{\partial \hat{\mathcal{L}}}{\partial Q_{B ; \alpha}}-\bar{D}_{\beta}\left(\frac{\partial \hat{\mathcal{L}}}{\partial Q_{B ; \alpha \beta}}\right)\right] Q_{B}\right|_{\sigma} ^{\tau} \\
& -\frac{\partial \hat{\mathcal{L}}}{\partial Q_{B ; \tau \alpha}} \bar{D}_{\sigma} Q_{B}+\frac{\partial \hat{\mathcal{L}}}{\partial Q_{B ; \beta \alpha}} \bar{D}_{\beta}\left(\left.Q_{B}\right|_{\sigma} ^{\tau}\right) .
\end{aligned}
$$

To derive $u$ in (38) we have already (40) with (41), and (43). We need to represent only (10), where the first two terms are evidently covariant (see (29) and (35)); the third term is defined by (42) and by (27):

$$
-\frac{\delta \hat{L}}{\delta Q_{B, \alpha}} \partial_{\sigma} Q_{B}=-\frac{\delta \hat{L}}{\delta Q_{B, \alpha}}\left(\bar{D}_{\sigma} Q_{B}-\left.\bar{\Gamma}_{\rho \sigma}^{\tau} Q_{B}\right|_{\tau} ^{\rho}\right)
$$

and the fourth term is defined by (33) and (28)

$$
\begin{aligned}
-\frac{\partial \hat{L}}{\partial Q_{B, \beta \alpha}} \partial_{\beta \sigma} Q_{B}= & -\frac{\partial \hat{\mathcal{L}}}{\partial Q_{B ; \beta \alpha}}\left(\bar{D}_{\beta \sigma} Q_{B}-\left.\bar{\Gamma}_{\sigma \rho, \beta}^{\tau} Q_{B}\right|_{\tau} ^{\rho}-\bar{\Gamma}_{\sigma \rho}^{\tau} \bar{D}_{\beta}\left(\left.Q_{B}\right|_{\tau} ^{\rho}\right)\right. \\
& \left.-\left.\bar{\Gamma}_{\beta \rho}^{\tau}\left(Q_{B ; \sigma}\right)\right|_{\tau} ^{\rho}+\left.\left.\bar{\Gamma}_{\sigma \rho}^{\tau} \bar{\Gamma}_{\beta \nu}^{\mu} Q_{B}\right|_{\tau} ^{\rho}\right|_{\mu} ^{\nu}\right) .
\end{aligned}
$$

Finally one has the evidently covariant form:

$$
\begin{aligned}
\hat{u}_{\sigma}{ }^{\alpha} & \equiv \hat{\mathcal{L}} \delta_{\sigma}^{\alpha}+\left.\frac{\delta \hat{\mathcal{L}}}{\delta Q_{B}} Q_{B}\right|_{\sigma} ^{\alpha}-\left[\frac{\partial \hat{\mathcal{L}}}{\partial Q_{B ; \alpha}}-\bar{D}_{\beta}\left(\frac{\partial \hat{\mathcal{L}}}{\partial Q_{B ; \alpha \beta}}\right)\right] \bar{D}_{\sigma} Q_{B} \\
& -\frac{\partial \hat{\mathcal{L}}}{\partial Q_{B ; \beta \alpha}} \bar{D}_{\beta \sigma} Q_{B}+\left.\frac{1}{2} \frac{\partial \hat{\mathcal{L}}}{\partial Q_{B ; \tau \alpha}} Q_{B}\right|_{\lambda} ^{\beta} \bar{R}_{\sigma \tau \beta}^{\lambda} .
\end{aligned}
$$

Showing that the coefficients in (37) (rewritten (18)) are covariant, we demonstrate that the expression under divergence in (37) in whole is a vector density, and the identity (37) can be rewritten as

$$
\bar{D}_{\alpha}\left[\hat{u}_{\sigma}{ }^{\alpha} \xi^{\sigma}+\hat{m}_{\sigma}{ }^{\alpha \tau} \bar{D}_{\tau} \xi^{\sigma}+\hat{n}_{\sigma}{ }^{\alpha \tau \beta} \bar{D}_{\beta \tau} \xi^{\sigma}\right] \equiv 0
$$

Opening it and equating independently to zero the coefficients at $\xi^{\sigma}, \bar{D}_{\alpha} \xi^{\sigma}$, $\bar{D}_{(\beta \alpha)} \xi^{\sigma}$ and $\bar{D}_{(\gamma \beta \alpha)} \xi^{\sigma}$, we get a set of identities:

$$
\begin{aligned}
& \bar{D}_{\alpha} \hat{u}_{\sigma}{ }^{\alpha}+\frac{1}{2} \hat{m}_{\lambda}{ }^{\alpha \rho} \bar{R}_{\sigma \rho \alpha}^{\lambda}+\frac{1}{3} \hat{n}_{\lambda}{ }^{\alpha \rho \gamma} \bar{D}_{\gamma} \bar{R}_{\sigma \rho \alpha}^{\lambda} \equiv 0, \\
& \hat{u}_{\sigma}{ }^{\alpha}+\bar{D}_{\lambda} \hat{m}_{\sigma}{ }^{\lambda \alpha}+\hat{n}_{\lambda}{ }^{\tau \alpha \rho} \bar{R}_{\sigma \rho \tau}^{\lambda}+\frac{2}{3} \hat{n}_{\sigma}{ }^{\lambda \tau \rho} \bar{R}_{\tau \rho \lambda}^{\alpha} \equiv 0, \\
& \hat{m}_{\sigma}{ }^{(\alpha \beta)}+\bar{D}_{\lambda} \hat{n}_{\sigma}{ }^{\lambda(\alpha \beta)} \equiv 0 \\
& \hat{n}_{\sigma}^{(\alpha \beta \gamma)} \equiv 0 .
\end{aligned}
$$


Substituting here the initial definitions (38) - (40) one can be convinced that the system (50) - (52) consists of linear combinations of the Klein identities (14) - (16). The identity (49) corresponds to $\partial_{\alpha} \hat{U}_{\sigma}^{\alpha} \equiv 0$. The last is a consequence of (14) - (16). Analogously, (49) is not independent - it is a consequence of (50) - (52).

Since the equality (48) is identically satisfied, the current

$$
\hat{\imath}^{\alpha}=-\left[\hat{u}_{\sigma}{ }^{\alpha} \xi^{\sigma}+\hat{m}_{\sigma}{ }^{\alpha \tau} \bar{D}_{\tau} \xi^{\sigma}+\hat{n}_{\sigma}{ }^{\alpha \tau \beta} \bar{D}_{\beta \tau} \xi^{\sigma}\right]
$$

must be a divergence of a superpotential (antisymmetrical tensor density):

$$
\hat{\imath}^{\alpha} \equiv \partial_{\beta} \hat{\imath}^{\alpha \beta}
$$

for which $\partial_{\beta \alpha} \hat{\imath}^{\alpha \beta} \equiv 0$. Indeed, substituting $\hat{u}_{\sigma}{ }^{\alpha}$ from (50) into the current (53), using (51) and algebraic properties of $\hat{n}_{\sigma}{ }^{\alpha \beta \gamma}$ and $\bar{R}_{\beta \rho \sigma}^{\alpha}$, and conserving the covariance, we reconstruct (53) into the form (54), where the superpotential is

$$
\hat{\imath}^{\alpha \beta}=\left(\frac{2}{3} \bar{D}_{\lambda} \hat{n}_{\sigma}^{[\alpha \beta] \lambda}-\hat{m}_{\sigma}^{[\alpha \beta]}\right) \xi^{\sigma}-\frac{4}{3} \hat{n}_{\sigma}^{[\alpha \beta] \lambda} \bar{D}_{\lambda} \xi^{\sigma} .
$$

It is explicitly antisymmetric in $\alpha$ and $\beta$.

At last, the current in (53) can be rewritten as

$$
\hat{\imath}^{\alpha}=-\left[\left(\hat{u}_{\sigma}^{\alpha}+\hat{n}_{\lambda}^{\alpha \beta \gamma} \bar{R}_{\beta \gamma \sigma}^{\lambda}\right) \xi^{\sigma}+\hat{m}^{\rho \alpha \beta} \partial_{[\beta} \xi_{\rho]}+\hat{z}^{\alpha}\right]
$$

where $z$-term is defined as

$$
\hat{z}^{\alpha}(\xi)=\hat{m}^{\sigma \alpha \beta} \zeta_{\sigma \beta}+\hat{n}^{\rho \alpha \beta \gamma}\left(2 \bar{D}_{\gamma} \zeta_{\beta \rho}-\bar{D}_{\rho} \zeta_{\beta \gamma}\right)
$$

and $2 \zeta_{\rho \sigma}=-£_{\xi} \bar{g}_{\rho \sigma}=2 \bar{D}_{(\rho} \xi_{\sigma)}$. Thus, $z$-term disappears, if $\xi^{\mu}$ is a Killing vector of the background spacetime. Then only the current (56) is determined by the energy-momentum $(u+n \bar{R})$-term and the spin $m$-term.

Now, let us sum the results. Instead of the non-covariant coefficients (10), (11) and (12), correspondent covariant coefficients (41), (44) and (47) have been constructed. Instead of the non-covariant Klein-Nœether identities (14) (16), (17), correspondent covariant identities (50) - (52), (36) are presented. By the construction, the explicitly covariant current (53) is equal to the current (21) in the original form exactly: $\hat{\imath}^{\alpha} \equiv \hat{I}^{\alpha}$. However, one can show that

$$
\hat{\imath}^{\alpha \beta}=\hat{I}^{\alpha \beta}+\frac{4}{3} \partial_{\lambda}\left(\hat{N}_{\sigma}^{[\lambda \beta] \alpha} \xi^{\sigma}\right)
$$

This means, of course, that $\partial_{\beta} \hat{\imath}^{\alpha \beta} \equiv \partial_{\beta} \hat{I}^{\alpha \beta}$ for the superpotentials (55) and (23), thus there is no a contradiction. 


\subsection{Another variant of covariantization}

In Sect. 2, all the identities and conserved quantities are derived through partial derivatives. The order of partial derivatives is not important because they are symmetrical with respect to replacements. For example, expressions, like $\partial \hat{L} / \partial Q_{B, \alpha \beta}$, are symmetrical in $\alpha$ and $\beta$. Nevertheless, in previous subsection, we did not used the symmetry of partial derivatives, conserving an original order of derivatives in the identities (unlike [24]), see remark after (33)). This has permitted us to present the covariant versions of identities and conserved quantities. However, there are another possibilities.

To introduce the situation let us consider an auxiliary Lagrangian $\hat{L}^{\text {test }}=$ $\hat{P}^{B \alpha \beta} Q_{B, \alpha \beta}+\ldots$ as an example. After direct covariantization it acquires the form $\hat{\mathcal{L}}^{t e s t}=\hat{P}^{B \alpha \beta} Q_{B ; \alpha \beta}+\ldots$. The variation with respect to $Q_{B, \alpha \beta}$ in the fist case gives $P^{B(\alpha \beta)}$. However, originally $P^{B \alpha \beta}$ is not necessarily symmetrical in $\alpha$ and $\beta$, therefore in the second case the variation with respect to $Q_{B ; \alpha \beta}$ gives simply $P^{B \alpha \beta}$. Thus, unlike the first case, the other order of second covariant derivatives can lead to a different result. If we symmetrize $\alpha$ and $\beta$ in the second case: $\hat{\mathcal{L}}^{\text {test }}=\hat{P}^{B \alpha \beta} Q_{B ;(\alpha \beta)}+\ldots$ then we need to change the other terms in the Lagrangian.

To study the problem of a different order of second covariant derivatives let us change this order in (29):

$$
\begin{aligned}
\hat{L}\left(Q_{B}, Q_{B, \alpha}, Q_{B, \alpha \beta}\right) & \equiv \hat{\mathcal{L}}\left(Q_{B}, Q_{B ; \alpha}, Q_{B ; \alpha \beta}, \bar{g}_{\mu \nu}, \bar{R}_{\mu \beta \nu}^{\alpha}\right) \\
& \equiv \hat{\mathcal{L}}\left(Q_{B}, Q_{B ; \alpha}, Q_{B ; \beta \alpha}+\left.Q_{B}\right|_{\sigma} ^{\rho} \bar{R}_{\rho}{ }^{\sigma}{ }_{\alpha \beta}, \bar{g}_{\mu \nu}, \bar{R}_{\mu \beta \nu}^{\alpha}\right) \\
& \equiv \hat{\mathcal{L}}^{*}\left(Q_{B}, Q_{B ; \alpha}, Q_{B ; \alpha \beta}, \bar{g}_{\mu \nu}, \bar{R}_{\mu \beta \nu}^{\alpha}\right) .
\end{aligned}
$$

For the sake of clearance one has to explain the notations. Here, the second line has the form of the Lagrangian of the first line, only the inverse second covariant derivatives are used. One can see that the arguments are mixed at the second line. After re-ordering the arguments, following the first line, it is clear that the Lagrangian acquires the other form (the third line). The star form is useful for the presentation because we need not remark every time that we use the inverse order of derivatives.

After the exchange in (59), it is evidently that derivatives with respect to second covariant derivatives of $Q_{B}$ change their order, also one obtains an additional derivative with respect to $Q_{B}$, proportional to the Riemannian tensor. At first, one has to be convinced that after this exchange the equations of motion (7) do not change. Of course, for the starred Lagrangian the form of the Lagrangian derivative has to be the same (35). Then, substituting the second line of (59) into (35) one obtains

$\frac{\delta \hat{\mathcal{L}}^{*}}{\delta Q_{B}}=\frac{\partial \hat{\mathcal{L}}}{\partial Q_{B}}-\bar{D}_{\tau}\left(\frac{\partial \hat{\mathcal{L}}}{\partial Q_{B ; \tau}}\right)+\bar{D}_{\tau \beta}\left(\frac{\partial \hat{\mathcal{L}}}{\partial Q_{B ; \beta \tau}}\right)+\frac{\partial \hat{\mathcal{L}}}{\partial Q_{C ; \tau \beta}} \frac{\partial}{\partial Q_{B}}\left(\left.Q_{C}\right|_{\lambda} ^{\rho} \bar{R}_{\rho}{ }^{\lambda} \tau \beta\right)$.

Changing the order of derivatives in the third term at the right hand side, using (167) and other formulae in Appendix A, one can see that, indeed, it 
is same Lagrangian derivative (35). However, the different definitions of the covariantized Lagrangin in (59) lead to different conserved quantities that we show below.

The use of the second line of (59) in (41), (44) and (47) gives

$$
\begin{aligned}
\hat{n}_{\sigma}^{* \alpha \tau \beta} & \equiv \frac{1}{2}\left[\left.\frac{\partial \hat{\mathcal{L}}}{\partial Q_{B ; \alpha \beta}} Q_{B}\right|_{\sigma} ^{\tau}+\left.\frac{\partial \hat{\mathcal{L}}}{\partial Q_{B ; \alpha \tau}} Q_{B}\right|_{\sigma} ^{\beta}\right], \\
\hat{m}_{\sigma}^{* \alpha \tau} & \left.\equiv\left[\frac{\partial \hat{\mathcal{L}}}{\partial Q_{B ; \alpha}}-\bar{D}_{\beta}\left(\frac{\partial \hat{\mathcal{L}}}{\partial Q_{B ; \beta \alpha}}\right)\right] Q_{B}\right|_{\sigma} ^{\tau}-\frac{\partial \hat{\mathcal{L}}}{\partial Q_{B ; \alpha \tau}} \bar{D}_{\sigma} Q_{B} \\
& +\frac{\partial \hat{\mathcal{L}}}{\partial Q_{B ; \alpha \beta}} \bar{D}_{\beta}\left(\left.Q_{B}\right|_{\sigma} ^{\tau}\right), \\
\hat{u}_{\sigma}^{* \alpha} & \equiv \hat{\mathcal{L}} \delta_{\sigma}^{\alpha}+\left.\frac{\delta \hat{\mathcal{L}}}{\delta Q_{B}} Q_{B}\right|_{\sigma} ^{\alpha}-\left[\frac{\partial \hat{\mathcal{L}}}{\partial Q_{B ; \alpha}}-\bar{D}_{\beta}\left(\frac{\partial \hat{\mathcal{L}}}{\partial Q_{B ; \beta \alpha}}\right)\right] \bar{D}_{\sigma} Q_{B} \\
& -\frac{\partial \hat{\mathcal{L}}}{\partial Q_{B ; \alpha \beta}} \bar{D}_{\beta \sigma} Q_{B}+\left.\frac{1}{2} \frac{\partial \hat{\mathcal{L}}}{\partial Q_{B ; \alpha \tau}} Q_{B}\right|_{\lambda} ^{\beta} \bar{R}_{\sigma \tau \beta}^{\lambda} .
\end{aligned}
$$

Remark, to obtain the set (61) - (63) one has to turn to the expressions (41), (44) and (47) and change the order of the second derivatives in all the terms, like $\partial \hat{\mathcal{L}} / \partial Q_{B ; \alpha \beta} \rightarrow \partial \hat{\mathcal{L}} / \partial Q_{B ; \beta \alpha}$, simultaneously.

Because all the expressions (61) - (63) have been obtained from the Lagrangian $\hat{\mathcal{L}}^{*}$ that is a scalar density, like $\hat{\mathcal{L}}$, they have to satisfy all the same identities (49) - (52) also. Let us show this. It is not difficult to find a connection of the expressions (61) - (63) with the coefficients (41), (44) and (47):

$$
\begin{aligned}
\hat{n}_{\sigma}^{* \alpha \tau \beta} & \equiv \hat{n}_{\sigma}{ }^{\alpha \tau \beta}+\left.\frac{\partial \hat{\mathcal{L}}}{\partial Q_{B ;[\alpha \beta]}} Q_{B}\right|_{\sigma} ^{\tau}+\left.\frac{\partial \hat{\mathcal{L}}}{\partial Q_{B ;[\alpha \tau]}} Q_{B}\right|_{\sigma} ^{\beta} . \\
\hat{m}_{\sigma}^{* \alpha \tau} & \equiv \hat{m}_{\sigma}{ }^{\alpha \tau}-2 \frac{\partial \hat{\mathcal{L}}}{\partial Q_{B ;[\alpha \tau]}} \bar{D}_{\sigma} Q_{B}+2 \bar{D}_{\beta}\left(\left.\frac{\partial \hat{\mathcal{L}}}{\partial Q_{B ;[\alpha \beta]}} Q_{B}\right|_{\sigma} ^{\tau}\right) . \\
\hat{u}_{\sigma}^{* \alpha} & \equiv \hat{u}_{\sigma}{ }^{\alpha}-2 \bar{D}_{\beta}\left(\frac{\partial \hat{\mathcal{L}}}{\partial Q_{B ;[\alpha \beta]}} \bar{D}_{\sigma} Q_{B}\right)+\left.\frac{\partial \hat{\mathcal{L}}}{\partial Q_{B ;[\alpha \tau]}} Q_{B}\right|_{\lambda} ^{\beta} \bar{R}_{\sigma \tau \beta}^{\lambda}
\end{aligned}
$$

where we define

$$
\frac{\partial \hat{\mathcal{L}}}{\partial Q_{B ;[\alpha \beta]}} \equiv \frac{1}{2}\left(\frac{\partial \hat{\mathcal{L}}}{\partial Q_{B ; \alpha \beta}}-\frac{\partial \hat{\mathcal{L}}}{\partial Q_{B ; \beta \alpha}}\right) .
$$

A direct substitution of (64) - (66) into the identities (49) - (52) shows that $n^{*}, m^{*}$ and $u^{*}$ satisfy them also, like the coefficients $n, m$ and $u$. This means that if we construct a starred current with using the rule (53):

$$
\hat{\imath}^{* \alpha}=-\left[\hat{u}_{\sigma}^{* \alpha} \xi^{\sigma}+\hat{m}_{\sigma}^{* \alpha \tau} \bar{D}_{\tau} \xi^{\sigma}+\hat{n}_{\sigma}^{* \alpha \tau \beta} \bar{D}_{\beta \tau} \xi^{\sigma}\right],
$$


then it is conserved identically. Indeed, it is easily to find that

$$
\hat{\imath}^{* \alpha}=\hat{\imath}^{\alpha}-2 \bar{D}_{\beta}\left(\frac{\partial \hat{\mathcal{L}}}{\partial Q_{B ;[\alpha \beta]}} £_{\xi} Q_{B}\right) .
$$

Then $\partial_{\alpha} \hat{\imath}^{* \alpha} \equiv \partial_{\alpha} \hat{\imath}^{\alpha}$, and consequently $\partial_{\alpha} \hat{\imath}^{* \alpha} \equiv 0$. Analogously to (54), the identity

$$
\hat{\imath}^{* \alpha} \equiv \partial_{\beta} \hat{\imath}^{* \alpha \beta}
$$

exists where

$$
\hat{\imath}^{* \alpha \beta}=\left(\frac{2}{3} \bar{D}_{\lambda} \hat{n}_{\sigma}^{*[\alpha \beta] \lambda}-\hat{m}_{\sigma}^{*[\alpha \beta]}\right) \xi^{\sigma}-\frac{4}{3} \hat{n}_{\sigma}^{*[\alpha \beta] \lambda} \bar{D}_{\lambda} \xi^{\sigma} .
$$

The direct substitution of (64) and (65) into (71) gives

$$
\begin{aligned}
\hat{\imath}^{* \alpha \beta} & =\hat{\imath}^{\alpha \beta}-2 \frac{\partial \hat{\mathcal{L}}}{\partial Q_{B ;[\alpha \beta]}} £_{\xi} Q_{B} \\
& +\frac{2}{3} \bar{D}_{\rho}\left[\xi^{\sigma}\left(\left.\frac{\partial \hat{\mathcal{L}}}{\partial Q_{B ;[\alpha \beta]}} Q_{B}\right|_{\sigma} ^{\rho}-\left.\frac{\partial \hat{\mathcal{L}}}{\partial Q_{B ;[\alpha \rho]}} Q_{B}\right|_{\sigma} ^{\beta}+\left.\frac{\partial \hat{\mathcal{L}}}{\partial Q_{B ;[\beta \rho]}} Q_{B}\right|_{\sigma} ^{\alpha}\right)\right] .
\end{aligned}
$$

The expression in the square brackets is antisymmetric in $\alpha, \beta$ and $\rho$. Thus $\bar{D}_{\rho}$ can be changed by $\partial_{\rho}$ and one can see that the term in the square brackets does not contribute into the current in (70). Also, due to the Stockes theorem this term does not contribute into surface integrals calculated with the use of the superpotential. Therefore we use only

$$
\hat{\imath}^{* \alpha \beta}=\hat{\imath}^{\alpha \beta}-2 \frac{\partial \hat{\mathcal{L}}}{\partial Q_{B ;[\alpha \beta]}} £_{\xi} Q_{B},
$$

which is in a correspondence with (69) and (70).

3.3 A new family of the covariant Nœther identically conserved quantities

It is worthy to discuss the situation. One has the identities

$$
\partial_{\alpha} \hat{I}^{\alpha} \equiv \partial_{\alpha} \hat{\imath}^{\alpha} \equiv \partial_{\alpha} \hat{\imath}^{* \alpha} \equiv 0 .
$$

Recall that each of the Lagrangains $\hat{L}, \hat{\mathcal{L}}$ and $\hat{\mathcal{L}}^{*}$ gives the same equations of motion for $Q_{B}$. Then adding (17) in a related form to each of the identities in (74) one obtains

$$
\begin{aligned}
&-\frac{\delta \hat{L}}{\delta Q_{B}} Q_{B, \alpha}-\partial_{\beta}\left(\left.\frac{\delta \hat{L}}{\delta Q_{B}} Q_{B}\right|_{\alpha} ^{\beta}\right)-\partial_{\alpha} \hat{I}^{\alpha} \equiv 0, \\
&-\frac{\delta \hat{\mathcal{L}}}{\delta Q_{B}} Q_{B ; \alpha}-\bar{D}_{\beta}\left(\left.\frac{\delta \hat{\mathcal{L}}}{\delta Q_{B}} Q_{B}\right|_{\alpha} ^{\beta}\right)-\partial_{\alpha} \hat{\imath}^{\alpha} \equiv 0 \\
&-\frac{\delta \hat{\mathcal{L}}^{*}}{\delta Q_{B}} Q_{B ; \alpha}-\bar{D}_{\beta}\left(\left.\frac{\delta \hat{\mathcal{L}}^{*}}{\delta Q_{B}} Q_{B}\right|_{\alpha} ^{\beta}\right)-\partial_{\alpha} \hat{\imath}^{* \alpha} \equiv 0 .
\end{aligned}
$$


Each of these identities is its own form of the unique identity (9). All of the identities $(75)$ - (77) can be interpreted as following after variation of the same Lagrangian. Therefore, the choice of both $\hat{\imath}^{\alpha}$ and $\hat{\imath}^{* \alpha}$, as a covariantized current, has equal rights. Nevertheless, what could be preferable from them? From the first glance it seems that it is $\hat{\imath}^{\alpha}$ because by the construction, $\hat{\imath}^{\alpha} \equiv \hat{I}^{\alpha}$. On the other hand, a conservation of the symmetry of partial derivatives looks as a nice idea. Then one can choose $\hat{L}\left(Q_{B}, Q_{B, \alpha}, Q_{B, \alpha \beta}\right) \equiv$ $\hat{\mathcal{L}}\left(Q_{B}, Q_{B ; \alpha}, Q_{B ;(\alpha \beta)}+\left.\frac{1}{2} Q_{B}\right|_{\sigma} ^{\rho} \bar{R}_{\rho}^{\sigma}{ }_{\alpha \beta}, \bar{g}_{\mu \nu}, \bar{R}_{\mu \beta \nu}^{\alpha}\right)$ instead of (59). In a reality, we do not see any theoretical foundation for a choice. Possibly, in future, applications to complicated solutions of the numerous modern modifications of GR will permit to do the choice. To unite aforementioned possibilities for constructing covariant conserved quantities we suggest a covariantized Lagrangian of the form:

$$
\begin{aligned}
\hat{L}\left(Q_{B}, Q_{B, \alpha}, Q_{B, \alpha \beta}\right) & \equiv \hat{\mathcal{L}}^{\dagger}\left(Q_{B}, Q_{B ; \alpha}, Q_{B ; \alpha \beta}, \bar{g}_{\mu \nu}, \bar{R}_{\mu \beta \nu}^{\alpha}\right) \\
& \equiv p \hat{\mathcal{L}}\left(Q_{B}, Q_{B ; \alpha}, Q_{B ; \alpha \beta}, \bar{g}_{\mu \nu}, \bar{R}_{\mu \beta \nu}^{\alpha}\right) \\
& +q \hat{\mathcal{L}}^{*}\left(Q_{B}, Q_{B ; \alpha}, Q_{B ; \alpha \beta}, \bar{g}_{\mu \nu}, \bar{R}_{\mu \beta \nu}^{\alpha}\right)
\end{aligned}
$$

where $p+q=1$ with real $p$ and $q$. The Lagrangian (78) leads to the same field equations (7), whereas the conservation law and conserved quantities for (78) are defined now as

$$
\begin{aligned}
\hat{\imath}^{\dagger \alpha} & \equiv \partial_{\beta} \hat{\imath}^{\dagger \alpha \beta}, \\
\hat{\imath}^{\dagger \alpha} & \equiv p \hat{\imath}^{\alpha}+q \hat{\imath}^{* \alpha}, \\
\hat{\imath}^{\dagger \alpha \beta} & \equiv p \hat{\imath}^{\alpha \beta}+q \hat{\imath}^{* \alpha \beta},
\end{aligned}
$$

presenting in a reality a family of identically conserved quantities.

To finalize subsection one has to note the following. Recall that a divergence in the Lagrangian, being non-essential for deriving field equations, is important (frequently even crucial) in a definition of Nother canonical conserved quantities. We give some necessary formulae. For the scalar density $\hat{\mathcal{L}}^{\prime}=\hat{d}^{\nu}{ }_{, \nu}$ one has the Nœether identity $\left(£_{\xi} \hat{d}^{\alpha}+\xi^{\alpha} \hat{d}^{\nu}{ }_{, \nu}\right)_{, \alpha} \equiv 0$, which has to be considered together with (4) (or (9)). This gives additional contributions into the current (53), also (69) or generalized (80), and into the superpotential $(55)$, also $(73)$ or generalized (81):

$$
\begin{aligned}
\hat{\imath}^{\prime \alpha} & =-\left[\hat{u}_{\sigma}^{\prime \alpha} \xi^{\sigma}+\hat{m}_{\sigma}^{\prime \alpha \tau} \bar{D}_{\tau} \xi^{\sigma}\right], \\
\hat{\imath}^{\prime \alpha \beta} & =-\hat{m}_{\sigma}^{\prime[\alpha \beta]} \xi^{\sigma}
\end{aligned}
$$

where

$$
\hat{u}_{\sigma}^{\alpha}=2 \bar{D}_{\beta}\left(\delta_{\sigma}^{[\alpha} \hat{d}^{\beta]}\right), \quad \hat{m}_{\sigma}^{\prime \alpha \beta}=2 \delta_{\sigma}^{[\alpha} \hat{d}^{\beta]}, \quad \hat{n}_{\sigma}^{\prime \alpha \beta \gamma}=0 .
$$

Note that a construction of these quantities does not depend on the inner structure of $\hat{d}^{\nu}$. 
3.4 The Belinfante corrected family of covariant identically conserved quantities

Here, we modify the results of previous subsections by the use of the Belinfante procedure [26]. Using the general Belinfante rule [13] we define a tensor density

$$
\hat{s}^{\alpha \beta \sigma} \equiv-\hat{s}^{\beta \alpha \sigma} \equiv-\hat{m}_{\lambda}{ }^{\sigma[\alpha} \bar{g}^{\beta] \lambda}-\hat{m}_{\lambda}^{\alpha[\sigma} \bar{g}^{\beta] \lambda}+\hat{m}_{\lambda}^{\beta[\sigma} \bar{g}^{\alpha] \lambda},
$$

which is called as a Belinfante correction, add $\bar{D}_{\beta}\left(\hat{s}^{\alpha \beta \sigma} \xi_{\sigma}\right)$ to both sides of (54) and obtain a new identity:

$$
\hat{\imath}_{B}^{\alpha} \equiv \partial_{\beta} \hat{\imath}_{B}^{\alpha \beta}
$$

This modification cancels the spin term from the current (56):

$$
\hat{\imath}_{B}^{\alpha} \equiv\left(-\hat{u}_{\sigma}{ }^{\alpha}-\hat{n}_{\lambda}{ }^{\alpha \beta \gamma} \bar{R}_{\beta \gamma \sigma}^{\lambda}+\bar{D}_{\beta} \hat{s}_{\sigma}^{\alpha \beta}\right) \xi^{\sigma}+\hat{z}_{B}^{\alpha}(\xi) \equiv \hat{u}_{B \sigma}{ }^{\alpha} \xi^{\sigma}+\hat{z}_{B}^{\alpha}(\xi)
$$

a new $z$-term disappears also on Killing vectors of the background:

$$
\begin{aligned}
\hat{z}_{B}^{\alpha}(\xi) & =\left(\hat{m}_{\lambda}^{\beta \alpha} \bar{g}^{\tau \lambda}+\hat{m}_{\lambda}{ }^{\alpha \tau} \bar{g}^{\beta \lambda}-\hat{m}_{\lambda}{ }^{\tau \beta} \bar{g}^{\alpha \lambda}\right) \zeta_{\tau \beta} \\
& +\hat{n}_{\lambda}{ }^{\alpha \tau \beta}\left(2 \bar{D}_{(\beta} \zeta_{\tau)}^{\lambda}-\bar{D}_{\sigma} \zeta_{\beta \tau} \bar{g}^{\lambda \sigma}\right) .
\end{aligned}
$$

Thus, the current $\hat{i}_{B}^{\alpha}$ is defined, in fact, by the modified energy-momentum tensor density $\hat{u}_{B \sigma}{ }^{\alpha}$. Because the new superpotential depends on the $n$-coefficients only:

$$
\hat{i}_{B}^{\alpha \beta} \equiv 2\left(\frac{1}{3} \bar{D}_{\rho} \hat{n}_{\sigma}^{[\alpha \beta] \rho}+\bar{D}_{\tau} \hat{n}_{\lambda}{ }^{\tau \rho[\alpha} \bar{g}^{\beta] \lambda} \bar{g}_{\rho \sigma}\right) \xi^{\sigma}-\frac{4}{3} \hat{n}_{\sigma}^{[\alpha \beta] \lambda} \bar{D}_{\lambda} \xi^{\sigma}
$$

then due to the definition (12) it vanishes for Lagrangians with only the first order derivatives. On the other hand, the superpotential (89) is well adapted to theories with second derivatives in Lagrangians, say, algebraically depending on Riemanninan tensor.

It is important to note that the Belinfante procedure cancels the contributions of the divergence into currents and superpotentials, see (82) - (84). Let us show this. The Belinfante correction constructed for the divergence by the rule (85) for (84) is presented as

$$
\hat{s}^{\prime \alpha \beta \sigma} \xi_{\sigma}=2 \xi^{[\alpha} \hat{d}^{\beta]} .
$$

Then, adding $\bar{D}_{\beta}\left(\hat{s}^{\alpha \beta \sigma} \xi_{\sigma}\right)$ and $\hat{s}^{\prime \alpha \beta \sigma} \xi_{\sigma}$ to (82) and (83) and keeping in mind (84) one obtains easily

$$
\begin{aligned}
\hat{\imath}^{\prime \alpha}+\bar{D}_{\beta}\left(\hat{s}^{\prime \alpha \beta \sigma} \xi_{\sigma}\right) & =-\left[\hat{u}_{\sigma}^{\prime \alpha} \xi^{\sigma}+\hat{m}_{\sigma}^{\prime \alpha \tau} \bar{D}_{\tau} \xi^{\sigma}\right]+\bar{D}_{\beta}\left(\hat{s}^{\prime \alpha \beta \sigma} \xi_{\sigma}\right)=0 ; \\
\hat{\imath}^{\prime \alpha \beta}+\hat{s}^{\prime \alpha \beta \sigma} \xi_{\sigma} & =-\hat{m}_{\sigma}^{\prime[\alpha \beta]} \xi^{\sigma}+\hat{s}^{\prime \alpha \beta \sigma} \xi_{\sigma}=0
\end{aligned}
$$

All the above can be applied exactly to the starred quantities in Subsect. 3.2 , obtaining in the result

$$
\hat{\imath}_{B}^{* \alpha} \equiv \partial_{\beta} \hat{\imath}_{B}^{* \alpha \beta}
$$


where

$$
\begin{aligned}
\hat{\imath}_{B}^{* \alpha} & \equiv\left(-\hat{u}_{\sigma}^{* \alpha}-\hat{n}_{\lambda}^{* \alpha \beta \gamma} \bar{R}_{\beta \gamma \sigma}^{\lambda}+\bar{D}_{\beta} \hat{s}_{\sigma}^{* \alpha \beta}\right) \xi^{\sigma}+\hat{z}_{B}^{* \alpha}(\xi), \\
\hat{i}_{B}^{* \alpha \beta} & \equiv 2\left(\frac{1}{3} \bar{D}_{\rho} \hat{n}_{\sigma}^{*[\alpha \beta] \rho}+\bar{D}_{\tau} \hat{n}_{\lambda}^{* \tau \rho[\alpha} \bar{g}^{\beta] \lambda} \bar{g}_{\rho \sigma}\right) \xi^{\sigma}-\frac{4}{3} \hat{n}_{\sigma}^{*[\alpha \beta] \lambda} \bar{D}_{\lambda} \xi^{\sigma} .
\end{aligned}
$$

The connection with the usual and starred Belinfante corrected quantities is

$$
\begin{aligned}
& \hat{\imath}_{B}^{* \alpha} \equiv \hat{\imath}_{B}^{\alpha}-2 \bar{D}_{\beta}\left[\left.\frac{\partial \hat{\mathcal{L}}}{\partial Q_{B ;[\alpha \beta]}} Q_{B}\right|_{\sigma} ^{\rho} \bar{D}_{\rho} \xi^{\sigma}+\bar{D}_{\rho}\left(\left.\frac{\partial \hat{\mathcal{L}}}{\partial Q_{B ;[\sigma \rho]}} Q_{B}\right|_{\lambda} ^{[\alpha} \bar{g}^{\beta] \lambda}\right.\right. \\
&\left.\left.+\left.\frac{\partial \hat{\mathcal{L}}}{\partial Q_{B ;[\alpha \rho]}} Q_{B}\right|_{\lambda} ^{[\sigma} \bar{g}^{\beta] \lambda}-\left.\frac{\partial \hat{\mathcal{L}}}{\partial Q_{B ;[\beta \rho]}} Q_{B}\right|_{\lambda} ^{[\sigma} \bar{g}^{\alpha] \lambda}\right) \xi_{\sigma}\right], \\
& \hat{\imath}_{B}^{* \alpha \beta} \equiv \hat{\imath}_{B}^{\alpha \beta}-2\left[\left.\frac{\partial \hat{\mathcal{L}}}{\partial Q_{B ;[\alpha \beta]}} Q_{B}\right|_{\sigma} ^{\rho} \bar{D}_{\rho} \xi^{\sigma}+\bar{D}_{\rho}\left(\left.\frac{\partial \hat{\mathcal{L}}}{\partial Q_{B ;[\sigma \rho]}} Q_{B}\right|_{\lambda} ^{[\alpha} \bar{g}^{\beta] \lambda}\right.\right. \\
&\left.\left.+\left.\frac{\partial \hat{\mathcal{L}}}{\partial Q_{B ;[\alpha \rho]}} Q_{B}\right|_{\lambda} ^{[\sigma} \bar{g}^{\beta] \lambda}-\left.\frac{\partial \hat{\mathcal{L}}}{\partial Q_{B ;[\beta \rho]}} Q_{B}\right|_{\lambda} ^{[\sigma} \bar{g}^{\alpha] \lambda}\right) \xi_{\sigma}\right] .
\end{aligned}
$$

The Belinfante corrected covariant conservation law and conserved quantities for the united covariantized Lagrangian (78) are defined as

$$
\begin{aligned}
\hat{\imath}_{B}^{\dagger \alpha} & \equiv \partial_{\beta} \hat{\imath}_{B}^{\dagger \alpha \beta}, \\
\hat{\imath}_{B}^{\dagger \alpha} & \equiv p \hat{\imath}_{B}^{\alpha}+q \hat{\imath}_{B}^{* \alpha}, \\
\hat{\imath}_{B}^{\dagger \alpha \beta} & \equiv p \hat{\imath}_{B}^{\alpha \beta}+q \hat{\imath}_{B}^{* \alpha \beta},
\end{aligned}
$$

presenting a Belinfante corrected family of identically conserved quantities.

It was remarked earlier that the conserved quantities constructed in $[1$, $20,21,23]$ are unique for the Lagrangian and in the framework of the Nother or of the Nœether-Belinfante procedure. There is no a contradiction with the results of this section where we suggest a new family of conserved quantities. It is because we have found here various possibilities to construct covariantized Lagrangians, in fact we suggest a family of such Lagrangians (78). Thus, for each of the Lagrangians of the family the conserved quantities are defined by an unique way.

\section{Conserved quantities for perturbations in arbitrary $D$-dimensional metric theories}

\subsection{A metric theory}

To present $D$-dimensional metric theory we consider the Lagrangian:

$$
\hat{L}_{D}=-\frac{1}{2 \kappa_{D}} \hat{L}_{g}\left(g_{\mu \nu}\right)+\hat{L}_{m}\left(g_{\mu \nu}, \Phi\right),
$$


which depends on the metric $g_{\mu \nu}$ and $\Phi$ and their derivatives up to a second order, where $\Phi$ defines matter sources without concretization. Thus $\hat{L}_{g}$ can be thought as an algebraic function of the metric and Riemannian tensors, $\hat{L}_{g}\left(g_{\mu \nu}\right)=\hat{L}_{g}\left(g_{\mu \nu}, R_{\rho \beta \sigma}^{\alpha}\right)$, that can be arbitrary. Variation of (101) with respect to $g^{\mu \nu}$ leads to the gravitational equations:

$$
\hat{\mathcal{G}}_{\mu \nu}=\kappa_{D} \hat{T}_{\mu \nu} .
$$

Variation of (101) with respect to $\Phi$ gives corresponding matter equations. Below we will use also the background Lagrangian defined as $\overline{\hat{L}}_{D}=\hat{L}_{D}\left(\bar{g}_{\mu \nu}, \bar{\Phi}\right)$ and corresponding background gravitational equations

$$
\overline{\hat{\mathcal{G}}}_{\mu \nu}=\kappa_{D} \overline{\hat{T}}_{\mu \nu}
$$

and matter equations. We set that the background fields $\bar{g}_{\mu \nu}$ and $\bar{\Phi}$ satisfy the background equations and, thus, are known (fixed).

In the present subsection, the subject of our attention is the gravitational part of the Lagrangian (101). Basing on the results of previous section, we set $Q^{A}=\left\{g_{\mu \nu}\right\}$ and incorporate an external metric $\bar{g}_{\mu \nu}$ into $\hat{L}_{g}$ in (101). A presentation of the Lagrangian in an "explicitly" covariant form with the use of $\bar{g}_{\mu \nu}$ is carried out exactly by the recipe of previous section. We change partial derivatives by covariant derivatives defined with respect to $\bar{g}_{\mu \nu}$. Thus, we transform the pure metric Lagrangian $\hat{L}_{g}$ into an explicitly covariant form:

$$
\hat{L}_{g}=\hat{\mathcal{L}}_{g}=\hat{\mathcal{L}}_{g}\left(g_{\mu \nu}, g_{\mu \nu ; \alpha}, g_{\mu \nu ; \alpha \beta}, \bar{g}_{\mu \nu}, \bar{R}_{\tau \rho \sigma}^{\lambda}\right) .
$$

Now we derive the coefficients (41), (44) and (47) for the Lagrangian $\hat{\mathcal{L}}=$ $-\hat{\mathcal{L}}_{g} / 2 \kappa_{D}$, setting there $Q^{A}=\left\{g_{\mu \nu}\right\}$. Thus, the coefficients are defined as follows. We directly rewrite $n$ and $m$ coefficients:

$$
\begin{gathered}
\hat{n}_{\sigma}{ }^{\alpha \tau \beta}=-\frac{1}{4 \kappa_{D}}\left[\left.\frac{\partial \hat{\mathcal{L}}_{g}}{\partial g_{\mu \nu ; \beta \alpha}} g_{\mu \nu}\right|_{\sigma} ^{\tau}+\left.\frac{\partial \hat{\mathcal{L}}_{g}}{\partial g_{\mu \nu ; \tau \alpha}} g_{\mu \nu}\right|_{\sigma} ^{\beta}\right] . \\
\hat{m}_{\sigma}{ }^{\alpha \tau}=-\frac{1}{2 \kappa_{D}}\left[\left.\frac{\delta \hat{\mathcal{L}}_{g}}{\delta g_{\mu \nu ; \alpha}} g_{\mu \nu}\right|_{\sigma} ^{\tau}-\frac{\partial \hat{\mathcal{L}}_{g}}{\partial g_{\mu \nu ; \tau \alpha}} \bar{D}_{\sigma} g_{\mu \nu}+\frac{\partial \hat{\mathcal{L}}_{g}}{\partial g_{\mu \nu ; \beta \alpha}} \bar{D}_{\beta}\left(\left.g_{\mu \nu}\right|_{\sigma} ^{\tau}\right)\right],
\end{gathered}
$$

where $\left.g_{\mu \nu}\right|_{\beta} ^{\alpha}=-2 g_{\beta(\mu} \delta_{\nu)}^{\alpha}$ and

$$
\frac{\delta \hat{\mathcal{L}}_{g}}{\delta g_{\mu \nu ; \alpha}} \equiv \frac{\partial \hat{\mathcal{L}}_{g}}{\partial g_{\mu \nu ; \alpha}}-\bar{D}_{\beta}\left(\frac{\partial \hat{\mathcal{L}}_{g}}{\partial g_{\mu \nu ; \alpha \beta}}\right) .
$$

It is useful to present $u$ coefficient in a structured form:

$$
\hat{u}_{\sigma}{ }^{\alpha}=-\frac{1}{\kappa_{D}}\left[\hat{\mathcal{G}}_{\sigma}^{\alpha}+\kappa_{D} \hat{\mathcal{U}}_{\sigma}{ }^{\alpha}+\kappa_{D} \hat{n}_{\lambda}{ }^{\alpha \tau \beta} \bar{R}_{\tau \beta \sigma}^{\lambda}\right],
$$


we use the notations

$$
\begin{aligned}
\hat{\mathcal{G}}_{\sigma}^{\alpha} & \left.\equiv \frac{1}{2} \frac{\delta \hat{\mathcal{L}}_{g}}{\delta g_{\mu \nu}} g_{\mu \nu}\right|_{\sigma} ^{\alpha} \equiv-\frac{\delta \hat{\mathcal{L}}_{g}}{\delta g_{\mu \alpha}} g_{\mu \sigma} \equiv \frac{\delta \hat{\mathcal{L}}_{g}}{\delta g^{\mu \sigma}} g^{\mu \alpha} \\
\hat{\mathcal{U}}_{\sigma}{ }^{\alpha} & \equiv-\frac{1}{2 \kappa_{D}}\left(\frac{\partial \hat{\mathcal{L}}_{g}}{\partial g_{\mu \nu ; \beta \alpha}} \bar{D}_{\sigma \beta} g_{\mu \nu}+\frac{\delta \hat{\mathcal{L}}_{g}}{\delta g_{\mu \nu ; \alpha}} \bar{D}_{\sigma} g_{\mu \nu}-\delta_{\sigma}^{\alpha} \hat{\mathcal{L}}_{g}\right) .
\end{aligned}
$$

As usual, $\delta \hat{\mathcal{L}}_{g} / \delta g_{\mu \nu}$ means Lagrangian derivatives, see $(35), \hat{\mathcal{G}}^{\alpha}{ }_{\sigma}$ is exactly the symmetrical left hand side of (102), and $\hat{\mathcal{U}}^{\alpha}{ }_{\sigma}$ is the generalized canonical energy-momentum related to the gravitational Lagrangian (104).

\subsection{Currents and superpotentials for perturbations}

Incorporation of the background metric is a key point, basing on which one has a possibility to describe perturbations. Perturbations are determined by the way when a one solution (dynamical) of the theory is considered as a perturbed system with respect to another solution (background) of the same theory. Then the background spacetime acquires a real sense, not auxiliary. Perturbations in such a derivation are exact (not infinitesimal or approximate), and then linear or of higher order approximations follow easily. We will denote $\delta$ as an exact difference between dynamical and background quantities:

$$
\delta Q^{A}=Q^{A}-\overline{Q^{A}} .
$$

Following to the Katz-Bičák-Lynden-Bell ideology [9] we construct the metric Lagrangian for perturbations:

$$
\hat{\mathcal{L}}_{G}=-\frac{1}{2 \kappa_{D}}\left(\hat{\mathcal{L}}_{g}-\overline{\hat{\mathcal{L}}}_{g}+\partial_{\alpha} \hat{d}^{\alpha}\right) .
$$

By the definition, the Lagrangian has to vanish for vanishing perturbations, therefore usually $\hat{d}^{\alpha}$ disappears for vanishing perturbations.

At first we construct the Nother canonical conserved quantities. Substituting (105), (106) and (108) into (53) (or to (56)), applying the barred procedure, subtracting one from another and taking into account the divergence in the way (82) - (84), one obtains the current corresponding to (112): $\delta \hat{\imath}^{\alpha}=\hat{\imath}^{\alpha}-\overline{\hat{\imath}^{\alpha}}+\hat{\imath}^{\prime \alpha}$. Then we use the dynamical equations (102) in $\hat{u}_{\sigma}{ }^{\alpha}$. We change $\hat{\mathcal{G}}_{\mu \nu}$ (as a part of $\hat{u}_{\sigma}{ }^{\alpha}$, see (108)) by the matter energy-momentum $\hat{T}_{\mu \nu}$ at the right hand side of (102). Next, we do the same combining $\overline{\hat{u}}_{\sigma}{ }^{\alpha}$ and the barred equations (103). In the result one obtains that the identically conserved current $\delta \hat{\imath}^{\alpha}$ related to (112) transforms into the current for perturbations:

$$
\hat{\mathcal{I}}^{\alpha}(\xi)=\hat{\Theta}_{\sigma}{ }^{\alpha} \xi^{\sigma}+\hat{\mathcal{M}}^{\sigma \alpha \beta} \partial_{[\sigma} \xi_{\beta]}+\hat{\mathcal{Z}}^{\alpha}(\xi) .
$$

Now, the conservation law:

$$
\partial_{\alpha} \hat{\mathcal{I}}^{\alpha}(\xi)=0
$$


takes a place due to the field equations, not identically. The generalized canonical energy-momentum tensor density, spin-term and $Z$-term for perturbations are

$$
\begin{aligned}
\hat{\Theta}_{\sigma}^{\alpha} & \equiv \delta \hat{T}_{\sigma}^{\alpha}+\delta \hat{\mathcal{U}}_{\sigma}^{\alpha}+\kappa_{D}^{-1} \bar{D}_{\beta}\left(\delta_{\sigma}^{[\alpha} \hat{d}^{\beta]}\right) \\
\hat{\mathcal{M}}^{\sigma \alpha \beta} & \equiv \delta \hat{m}_{\rho}^{\alpha \beta} \bar{g}^{\sigma \rho}-\kappa_{D}^{-1} \bar{g}^{\sigma[\alpha} \hat{d}^{\beta]} \\
\hat{\mathcal{Z}}^{\alpha}(\xi) & \equiv-\delta \hat{z}^{\alpha}+\kappa_{D}^{-1} \zeta_{\beta}^{[\alpha} \hat{d}^{\beta]}
\end{aligned}
$$

where $\delta$ has a sense of a general definition (111). To present the value of the current (113), one has to take solutions to the equations (102) and (103) and use them for calculating concrete values of the quantities (105), (106), (110) and (57). Starting from (55), by the same way we construct a superpotential corresponding to the current (113):

$$
\begin{aligned}
\delta \hat{\imath}^{\alpha \beta} & =\hat{\imath}^{\alpha \beta}-\overline{\hat{\imath}^{\alpha \beta}}+\hat{\imath}^{\alpha \beta} \rightarrow \\
\hat{\mathcal{I}}^{\alpha \beta} & =\left(\frac{2}{3} \bar{D}_{\lambda} \delta \hat{n}_{\sigma}{ }^{[\alpha \beta] \lambda}-\delta \hat{m}_{\sigma}{ }^{[\alpha \beta]}-2 \kappa_{D}^{-1} \delta_{\sigma}^{[\alpha} \hat{d}^{\beta]}\right) \xi^{\sigma}-\frac{4}{3} \delta \hat{n}_{\sigma}{ }^{[\alpha \beta] \lambda} \bar{D}_{\lambda} \xi^{\sigma}(118)
\end{aligned}
$$

Then, instead of (114) one can use the conservation law in the form:

$$
\hat{\mathcal{I}}^{\alpha}(\xi)=\partial_{\alpha} \hat{\mathcal{I}}^{\alpha \beta}(\xi) .
$$

It is not identity, but the conservation law for perturbations determined by the solutions to the equations (102) and (103).

Analogously the starred conservation law can be constructed. Thus, more generally, the family of the Nother canonical conservation laws for perturbations corresponding to the presentation (78) - (81) has a form:

$$
\hat{\mathcal{I}}^{\dagger \alpha}(\xi)=\partial_{\alpha} \hat{\mathcal{I}}^{\dagger \alpha \beta}(\xi) .
$$

It is edifying to find a connection of the generalized conserved quantities in (120) with known ones in 4D GR. Note, that even in D-dimensional GR there is no a difference between starred and non-starred quantities. It is because the Einstein part of (175) in Appendix B does not contain an antisymmetrical part. Therefore for GR the conservation law (120) is a single one, not a family. Thus, it is enough to take into account the Einstein part in (178) - (180) in Appendix B to derive a superpotential in (120) for GR. Next, choosing the Minkowski background in Cartesian coordinates and the translation Killing vectors in the form $\xi^{\alpha}=\delta_{(\beta)}^{\alpha}$ one recognizes that the superpotential in (120) goes to the very known Freud superpotential [37] in 4D GR.

To construct the Belinfante corrected conserved currents for the perturbed system (112) we substitute (105), (106) and (108) into (87) and subtract the corresponding barred expression (87): $\delta \hat{\imath}_{B}^{\alpha}=\hat{\imath}_{B}^{\alpha}-\overline{\hat{\imath}_{B}^{\alpha}}$. The same is obtained after applying the Nother-Belinfante method directly to the Lagrangian in (112). Again, using the equations (102) and their barred version (103) in $\hat{u}_{\sigma}{ }^{\alpha}$ and $\overline{\hat{u}}_{\sigma}{ }^{\alpha}$, the current $\delta \hat{\imath}_{B}^{\alpha}$ related to (112) transforms into

$$
\hat{\mathcal{I}}_{B}^{\alpha}(\xi)=\hat{\Theta}_{B \sigma}{ }^{\alpha} \xi^{\sigma}+\hat{\mathcal{Z}}_{B}^{\alpha}(\xi) .
$$


Thus, one has a conservation law for perturbations

$$
\partial_{\alpha} \hat{\mathcal{I}}_{B}^{\alpha}(\xi)=0,
$$

which takes a place on the field equations, not identically. Of course, the current (121) does not contain a spin term, unlike (113). The Belinfante corrected energy-momentum tensor density and $Z$-term for perturbations are

$$
\begin{aligned}
& \hat{\Theta}_{B \sigma}{ }^{\alpha} \equiv \delta \hat{T}_{\sigma}^{\alpha}+\delta \hat{\mathcal{U}}_{\sigma}{ }^{\alpha}+\bar{D}_{\beta} \delta \hat{s}^{\alpha \beta}{ }_{\sigma}, \\
& \hat{\mathcal{Z}}_{B}^{\alpha}(\xi) \equiv-\delta \hat{z}_{B}^{\alpha}(\xi) .
\end{aligned}
$$

Starting from (89), a superpotential corresponding to the current (121) is constructed analogously:

$$
\begin{aligned}
\delta \hat{\imath}_{B}^{\alpha \beta} & =\hat{\imath}_{B}^{\alpha \beta}-\overline{\hat{\imath}_{B}^{\alpha \beta}} \rightarrow \\
\hat{\mathcal{I}}_{B}^{\alpha \beta} & =\equiv 2\left(\frac{1}{3} \bar{D}_{\rho} \delta \hat{n}_{\sigma}^{[\alpha \beta] \rho}+\bar{D}_{\tau} \delta \hat{n}_{\lambda}{ }^{\tau \rho[\alpha} \bar{g}^{\beta] \lambda} \bar{g}_{\rho \sigma}\right) \xi^{\sigma}-\frac{4}{3} \delta \hat{n}_{\sigma}^{[\alpha \beta] \lambda} \bar{D}_{\lambda} \xi^{\sigma}(12
\end{aligned}
$$

Instead of (122) the conservation law for the perturbations can be presented also in the form:

$$
\hat{\mathcal{I}}_{B}^{\alpha}(\xi)=\partial_{\alpha} \hat{\mathcal{I}}_{B}^{\alpha \beta}(\xi) .
$$

It is not identity, all the quantities (105), (106), (110), also (85) and (88) are determined by the solutions to the equations (102) and (103).

By the same way the starred Belinfante corrected conservation law can be constructed. More generally, the family of the conservation laws corresponding to the presentation (78), (98) - (100) takes a place:

$$
\hat{\mathcal{I}}_{B}^{\dagger \alpha}(\xi)=\partial_{\alpha} \hat{\mathcal{I}}_{B}^{\dagger \alpha \beta}(\xi) .
$$

Again, for GR the conservation law (127) is a single one, not a family. Using the Einstein part in (181) and (182) in Appendix B one can be convinced that on the Minkowski background in Cartesian coordinates and with the translation Killing vectors the superpotential in (127) transforms into the well known Papapetrou superpotential [38] in 4D GR, see also [13].

\section{Applications}

\subsection{Conserved charges in EGB gravity}

In this section, we apply the results of previous sections to calculate mass of the Schwarzschild-anti-de Sitter (S-AdS) BH [27] in the EGB gravity. Reasons why we have chosen this solution are as follows. First, in the Einstein theory there are no differences between various conserved quantities of the new family (see discussion in previous section), but they are exist in the EGB gravity, which is a one of the most popular modifications of GR. Second, the S-AdS BH in the EGB gravity is a more known solution, which is frequently used as a standard solution in applications. 
Historically, in [20-23], it was suggested the starred variant of conservation laws for perturbations based on the identities (70) and (93) only. In in Appendix B, we present the necessary formulae for EGB gravity including the starred superpotentials (178) - (182) related to (71) and (95) on the right hand sides of (70) and (93). In [20,21], basing on these superpotentials, already we have obtained the mass for the aforementioned solution, which coincides exactly with the standard results. In previous sections, expanding the results of [20-23], we have suggested not only starred conserved quantities, but the family of conserved quantities united by (120) and (127). Here, we test all of them, calculating mass for the S-AdS BH in EGB gravity with the use of the generalized superpotentials in (120) and (127).

Rewrite the conservation laws of all the types for perturbations in the united form:

$$
\hat{\mathcal{I}}_{D}^{\alpha}(\xi)=\partial_{\beta} \hat{\mathcal{I}}_{D}^{\alpha \beta}(\xi)
$$

Here, a superpotential can be one of the set $\hat{\mathcal{I}}_{D}^{\alpha \beta}=\left\{\hat{\mathcal{I}}^{\dagger \alpha \beta}, \hat{\mathcal{I}}_{B}^{\dagger \alpha \beta}\right\}$; the same is related to the currents for perturbations. The conservation law (128) allows us to construct the conserved charges in generalized form in $D$-dimensions:

$$
\mathcal{P}(\xi)=\int_{\Sigma} d^{D-1} x \hat{\mathcal{I}}_{D}^{0}(\xi)=\oint_{\partial \Sigma} d S_{i} \hat{\mathcal{I}}_{D}^{0 i}(\xi)
$$

In next subsection we concretize the notations for a section $\Sigma$ and its boundary $\partial \Sigma$.

With the use of (81) and (100), (118) and (125), and starred (118) and (125) we represent the superpotentials in (120) and (127) in the form:

$$
\begin{aligned}
& \hat{\mathcal{I}}^{\dagger \alpha \beta}=\hat{\mathcal{I}}^{* \alpha \beta}+p\left[\left(\hat{\imath}^{\alpha \beta}-\hat{\imath}^{* \alpha \beta}\right)-\left(\overline{\hat{\imath}^{\alpha \beta}}-\overline{\hat{\imath}^{* \alpha \beta}}\right)\right]=\hat{\mathcal{I}}^{* \alpha \beta}+p \Delta \hat{\mathcal{I}}^{\alpha \beta}, \\
& \hat{\mathcal{I}}_{B}^{\dagger \alpha \beta}=\hat{\mathcal{I}}_{B}^{* \alpha \beta}+p\left[\left(\hat{\imath}_{B}^{\alpha \beta}-\hat{\imath}_{B}^{* \alpha \beta}\right)-\left(\overline{\hat{\imath}_{B}^{\alpha \beta}}-\overline{\hat{\imath}_{B}^{* \alpha \beta}}\right)\right]=\hat{\mathcal{I}}_{B}^{* \alpha \beta}+p \Delta \hat{\mathcal{I}}_{B}^{\alpha \beta} .
\end{aligned}
$$

The expressions in square brackets are defined by the formulae (73) and (97). Because for the S-AdS BH the starred superpotentials already have been checked we need only to calculate

$$
\Delta \mathcal{P}(\xi)=p \oint_{\partial \Sigma} d S_{i} \Delta \hat{\mathcal{I}}_{D}^{0 i}(\xi)
$$

where $\Delta \hat{\mathcal{I}}_{D}^{\alpha \beta}=\left\{\Delta \hat{\mathcal{I}}^{\alpha \beta}, \Delta \hat{\mathcal{I}}_{B}^{\alpha \beta}\right\}$.

To define $\Delta \hat{\mathcal{I}}_{D}^{\alpha \beta}$ in (130) and (131) for the EGB gravity one needs in an antisymmetrical in $\alpha$ and $\beta$ part of the expression (175), see (73) and (97). It is

$$
\frac{\partial \hat{\mathcal{L}}_{E G B}}{\partial g_{\mu \nu ;[\alpha \beta]}}=\frac{2 \alpha \sqrt{-g}}{\kappa_{D}}\left(R^{\alpha(\mu} g^{\nu) \beta}-R^{\beta(\mu} g^{\nu) \alpha}\right) .
$$


Thus, from (73) one has

$$
\begin{aligned}
\hat{\imath}^{\alpha \beta}-\hat{\imath}^{* \alpha \beta} & =2 \frac{\partial \hat{\mathcal{L}}_{E G B}}{\partial g_{\mu \nu ;[\alpha \beta]}} £_{\xi} g_{\mu \nu} \\
& =-\frac{8 \alpha \sqrt{-g}}{\kappa_{D}}\left(\bar{D}_{\mu} \xi^{\rho}+\xi^{\tau} \Delta_{\tau \mu}^{\rho}\right)\left(R_{\rho}^{[\alpha} g^{\beta] \mu}+R^{\mu[\alpha} \delta_{\rho}^{\beta]}\right) \\
& =-\frac{8 \alpha \sqrt{-g}}{\kappa_{D}}\left(R_{\rho}^{[\alpha} g^{\beta] \mu}+R^{\mu[\alpha} \delta_{\rho}^{\beta]}\right) D_{\mu} \xi^{\rho}
\end{aligned}
$$

where the quantities $\Delta_{\tau \mu}^{\rho}$ are presented by the relation (171) in Appendix B, and the definitions (172) and (173) are used also. From (97) one has

$$
\begin{aligned}
& \hat{\imath}_{B}^{\alpha \beta}-\hat{\imath}_{B}^{* \alpha \beta}=2\left[\left.\frac{\partial \hat{\mathcal{L}}}{\partial g_{\mu \nu ;[\alpha \beta]}} g_{\mu \nu}\right|_{\sigma} ^{\rho} \bar{D}_{\rho} \xi^{\sigma}+\bar{D}_{\rho}\left(\left.\frac{\partial \hat{\mathcal{L}}}{\partial g_{\mu \nu ;[\sigma \rho]}} g_{\mu \nu}\right|_{\lambda} ^{[\alpha} \bar{g}^{\beta] \lambda}\right.\right. \\
& \left.\left.+\left.\frac{\partial \hat{\mathcal{L}}}{\partial g_{\mu \nu ;[\alpha \rho]}} g_{\mu \nu}\right|_{\lambda} ^{[\sigma} \bar{g}^{\beta] \lambda}-\left.\frac{\partial \hat{\mathcal{L}}}{\partial g_{\mu \nu ;[\beta \rho]}} g_{\mu \nu}\right|_{\lambda} ^{[\sigma} \bar{g}^{\alpha] \lambda}\right) \xi_{\sigma}\right] \\
& =\frac{4 \alpha \sqrt{-g}}{\kappa_{D}}\left\{-2\left(R_{\sigma}^{[\alpha} g^{\beta] \rho}+R^{\rho[\alpha} \delta_{\sigma}^{\beta]}\right) \bar{D}_{\rho} \xi^{\sigma}\right. \\
& +\xi_{\sigma} \bar{D}_{\rho}\left[R_{\lambda}^{\sigma} g^{\rho[\alpha} \bar{g}^{\beta] \lambda}+g^{\sigma \rho} R_{\lambda}^{[\alpha} \bar{g}^{\beta] \lambda}-R_{\lambda}^{[\alpha} g^{\beta] \rho} \bar{g}^{\sigma \lambda}\right. \\
& \left.\left.+2\left(R^{\sigma[\alpha} \bar{g}^{\beta] \rho}-R^{\rho[\alpha} \bar{g}^{\beta] \sigma}-R_{\lambda}^{\rho} g^{\sigma[\alpha} \bar{g}^{\beta] \lambda}\right)\right]\right\} .
\end{aligned}
$$

The barred expressions are obtained easily.

5.2 Mass of the Schwarzschild-AdS black hole in EGB gravity

The S-AdS solution in EGB gravity (see formulae (168), (169) in Appendix B) has a form [27]:

$$
d s^{2}=-f d t^{2}+f^{-1} d r^{2}+r^{2} \sum_{a, b}^{D-2} q_{a b} d x^{a} d x^{b}
$$

with the metric components $g_{00}=-f(r)$ and $g_{11}=f^{-1}(r)$ where

$$
\begin{aligned}
f(r) & =1+\frac{r^{2}}{2 \alpha(D-3)(D-4)} \\
& \times\left\{1 \pm \sqrt{1-\frac{4 \Lambda_{0}}{\Lambda_{E G B}}+4 \alpha(D-3)(D-4) \frac{r_{0}^{D-3}}{r^{D-1}}}\right\}, \\
\Lambda_{E G B} & =-\frac{(D-2)(D-1)}{2 \alpha(D-4)(D-3)} .
\end{aligned}
$$


The last term in (136) describes $(D-2)$-dimensional sphere of the radius $r$, and $q_{a b}$ depends on coordinates on the sphere only. The Christoffel symbols corresponding (136) are

$\Gamma_{00}^{1}=\frac{f f^{\prime}}{2}, \quad \Gamma_{10}^{0}=\frac{f^{\prime}}{2 f}, \quad \Gamma_{11}^{1}=-\frac{f^{\prime}}{2 f}, \quad \Gamma_{1 b}^{a}=\frac{1}{r} \delta_{b}^{a}, \quad \Gamma_{a b}^{1}=-r f q_{a b}$

where 'prime' means $\partial / \partial r$. The Riemannian, Ricci tensors and curvature scalar corresponding to (136) are

$$
\begin{aligned}
R_{0101} & =\frac{1}{2} f^{\prime \prime}, \quad R_{0 a 0 b}=\frac{1}{2} r f f^{\prime} q_{a b}, \quad R_{1 a 1 b}=-\frac{r f^{\prime}}{2 f} q_{a b}, \\
R_{a b c d} & =-r^{2}(f-1)\left(q_{a c} q_{b d}-q_{a d} q_{b c}\right) ; \\
R_{00} & =\frac{f}{2}\left(f^{\prime \prime}+f^{\prime} \frac{D-2}{r}\right), \quad R_{11}=-\frac{1}{2 f}\left(f^{\prime \prime}+f^{\prime} \frac{D-2}{r}\right), \\
R_{a b} & =-\left[(f-1)(D-3)+r f^{\prime}\right] q_{a b} ; \\
R & =-\left(f^{\prime \prime}+f^{\prime} \frac{D-2}{r}\right)-\frac{D-2}{r^{2}}\left[(f-1)(D-3)+r f^{\prime}\right] .
\end{aligned}
$$

The barred solution (136) is defined by the condition $r_{0}=0$ and presents a background AdS solution with

$$
\bar{f}(r)=1-r^{2} \frac{2 \Lambda_{e f f}}{(D-1)(D-2)} .
$$

The effective cosmological constant is defined as

$$
\Lambda_{\text {eff }}=\frac{\Lambda_{E G B}}{2}\left(1 \pm \sqrt{1-\frac{4 \Lambda_{0}}{\Lambda_{E G B}}}\right),
$$

thus $\Lambda_{\text {eff }}$ is negative, see (138). For the metric (136) the relation $\sqrt{-g_{D}}=$ $\sqrt{-\bar{g}_{D}}=r^{D-2} \sqrt{\operatorname{det} q_{a b}}$ has a place and is important for calculations. The background Christoffel symbols are the barred expressions (139). The background Riemannian, Ricci tensors and curvature scalar are

$$
\bar{R}_{\mu \alpha \nu \beta}=2 \Lambda_{e f f} \frac{\left(\bar{g}_{\mu \nu} \bar{g}_{\alpha \beta}-\bar{g}_{\mu \beta} \bar{g}_{\nu \alpha}\right)}{(D-2)(D-1)}, \bar{R}_{\mu \nu}=2 \Lambda_{e f f} \frac{\bar{g}_{\mu \nu}}{D-2}, \bar{R}=2 \Lambda_{e f f} \frac{D}{D-2} .
$$

The barred expressions (140) go to (143). It is evidently that for solutions (136) perturbations can be described only by $\Delta f=f-\bar{f}$. Keeping only the first order term with respect to $1 / r$, we obtain

$$
\Delta f= \pm\left(\sqrt{1-\frac{4 \Lambda_{0}}{\Lambda_{E G B}}}\right)^{-1}\left(\frac{r_{0}}{r}\right)^{D-3} .
$$

In $[20,21]$, the mass of the S-AdS BH has been obtained with the use of the starred conserved quantities. The starred superpotentials (118) or (125) in 
the EGB gravity are presented by (178) - (182). Thus, using a timelike Killing vector $\xi^{\mu}=(-1, \mathbf{0})$ of the background and the S-AdS BH data (136) - (144), one obtains

$$
M=\lim _{r \rightarrow \infty} \oint_{\partial \Sigma} d x^{D-2} \sqrt{-\bar{g}_{D}} \mathcal{I}_{D}^{01}(\xi)=\frac{(D-2) r_{0}^{D-3}}{4 G_{D}} .
$$

Here, $\hat{\mathcal{I}}_{D}^{\alpha \beta}=\left\{\hat{\mathcal{I}}^{* \alpha \beta}, \hat{\mathcal{I}}_{B}^{* \alpha \beta}\right\} ; \partial \Sigma$ is the $(D-2)$ dimensional boundary of $\Sigma$ that is a spacelike $(D-1)$ hypersurface $x^{0}=$ const. The result $(145)$ is the standard accepted result obtained with using the various approaches (see, e.g., [14,17], [39-41] and references therein).

To examine the family of conservation laws (120) and (127), calculating the mass of the S-AdS BH and keeping in mind (145), it is enough to calculate the integral (132)

$$
\Delta M=p \lim _{r \rightarrow \infty} \oint_{\partial \Sigma} d x^{D-2} \sqrt{-\bar{g}_{D}} \Delta \mathcal{I}_{D}^{01}(\xi) .
$$

Here, the Killing vector $\xi^{\mu}=(-1, \mathbf{0})$ is used again, and (134) and (135) have to be calculated for (136) - (144). In the canonical Nœther case one obtains $\hat{\imath}^{\alpha \beta}-\hat{\imath}^{* \alpha \beta} \equiv 0$. The calculations for the Belinfante corrected case give

$$
\hat{\imath}_{B}^{\alpha \beta}-\hat{\imath}_{B}^{* \alpha \beta}=\frac{4 \alpha \sqrt{-\bar{g}}}{\kappa_{D}}\left(g^{00}-\bar{g}^{00}\right)\left(g^{a b} R_{0}^{0}-R^{a b}\right) \bar{\Gamma}_{a b}^{1} .
$$

The formulae (136) - (144) show that $\Delta \hat{\mathcal{I}}_{B}^{01}=\hat{\imath}_{B}^{\alpha \beta}-\hat{\imath}_{B}^{* \alpha \beta} \sim 1 / r^{D+1}$. Thus, for the canonical Nother case (120) $\Delta M=0$ identically, whereas for the Belinfante corrected case (127) $\Delta M=0$ due to the asymptotical behavior. This means that, calculating mass of the S-AdS BH in the EGB gravity, all the superpotentials of the new family give the same standard result (145).

\section{Concluding remarks}

In the paper, expanding possibilities for constructing conservation laws and conserved quantities for perturbations on arbitrary curved backgrounds in metric theories, we have suggested a new family of such expressions and quantities. Particular types of conservation laws, which relate to this family, already exist and have been applied. Thus, in [20-23], the conserved quantities denoted here as a starred ones have been presented, see Subsect. 3.2 and Appendix B. The quantities derived in [17] are related to the type considered in Subsect. 3.1. Let us show this.

Reformulating the superpotential (4.8) in the Deruelle, Katz and Ogushi paper [17] constructed in the EGB gravity in our notations, one obtains

$$
\hat{\imath}_{D K O}^{\alpha \beta}=\frac{\sqrt{-g}}{\kappa_{D}} D^{[\alpha} \xi^{\beta]}-\frac{2 \alpha \sqrt{-g}}{\kappa_{D}}\left\{R_{\sigma}^{\lambda \alpha \beta}+4 R^{\lambda[\alpha} \delta_{\sigma}^{\beta]}+\delta_{\sigma}^{[\alpha} g^{\beta] \lambda} R\right\} D_{\lambda} \xi^{\sigma} .
$$


Reformulating the starred superpotential (178) in the terms of the dynamical covariant derivative (172) with the use of (173), one obtains

$$
\hat{\imath}^{* \alpha \beta}=\frac{\sqrt{-g}}{\kappa_{D}} D^{[\alpha} \xi^{\beta]}-\frac{2 \alpha \sqrt{-g}}{\kappa_{D}}\left\{R_{\sigma}{ }^{\lambda \alpha \beta}+4 g^{\lambda[\alpha} R_{\sigma}^{\beta]}+\delta_{\sigma}^{[\alpha} g^{\beta] \lambda} R\right\} D_{\lambda} \xi^{\sigma} .
$$

Comparing $\hat{\imath}_{D K O}^{\alpha \beta}-\hat{\imath}^{* \alpha \beta}$ and the difference $\hat{\imath}^{\alpha \beta}-\hat{\imath}^{* \alpha \beta}$ presented in (134) one can see that $\hat{\imath}_{D K O}^{\alpha \beta}=\hat{\imath}^{\alpha \beta}$, where $\hat{\imath}^{\alpha \beta}$ is just the superpotential of the type constructed in subsect. 3.1. However, recall that an additional divergence in the Lagrangian in [17] differs from the divergence here defined by (180) in Appendix B, see remarks around (180).

The result of the application here is that all the superpotentials of the family give the same standard accepted mass for the S-AdS BH. Thus, differences between various conserved quantities of the family, possibly, look as not essential. However, numerus solutions of popular gravitational theories frequently have very exotic properties. Therefore, wider possibilities to study such solutions are desirable, and, in this relation, the suggested family presents a more universal instrument. We do not exclude the situation when any solution any modified theory of gravity could be a crucial test solution for a choice between members (conserved quantities) of the family. We plan such applications in future.

\section{A Auxiliary algebraic expressions}

Here, we give useful for calculations algebraic properties of the operator presented by the notation $\left.Q^{A}\right|_{\beta} ^{\alpha}$ included in (3):

$$
\delta Q^{A}=£_{\xi} Q^{A}=-\xi^{\alpha} \partial_{\alpha} Q^{A}+\left.Q^{A}\right|_{\beta} ^{\alpha} \partial_{\alpha} \xi^{\beta} .
$$

In general, we follow to [24], however our treating $\left.Q^{A}\right|_{\beta} ^{\alpha}$ is more simple and more effective, as we imagine. We define the operator for covariant quantities only: tensor densities or sets of tensor densities. For example, for a tensor density of the weight $+n$ one has

$$
\begin{aligned}
\left.Q_{\pi \rho \ldots \sigma}^{\alpha \beta \ldots \gamma}\right|_{\nu} ^{\mu} & =-n \delta_{\nu}^{\mu} Q_{\pi \rho \ldots \sigma}^{\alpha \beta \ldots \gamma}+\delta_{\nu}^{\alpha} Q_{\pi \rho \ldots \sigma}^{\mu \beta \ldots \gamma}+\delta_{\nu}^{\beta} Q_{\pi \rho \ldots \sigma}^{\alpha \mu \ldots \gamma}+\ldots+\delta_{\nu}^{\gamma} Q_{\pi \rho \ldots \sigma}^{\alpha \beta \ldots \mu} \\
& -\delta_{\pi}^{\mu} Q_{\nu \rho \ldots \sigma}^{\alpha \beta \ldots \gamma}-\delta_{\rho}^{\mu} Q_{\pi \nu \ldots \sigma}^{\alpha \beta \ldots \gamma}-\ldots-\delta_{\sigma}^{\mu} Q_{\pi \rho \ldots \nu}^{\alpha \beta \ldots \gamma} .
\end{aligned}
$$

Thus, for the metric one has $\left.g_{\mu \nu}\right|_{\beta} ^{\alpha}=-\delta_{\mu}^{\alpha} g_{\beta \nu}-\delta_{\nu}^{\alpha} g_{\mu \beta}$, or for the scalar density: $\left.\hat{\mathcal{L}}\right|_{\beta} ^{\alpha}=$ $-\delta_{\beta}^{\alpha} \hat{\mathcal{L}}$. One can see that calculations with expressions, like (151), could be very cumbersome. Whereas the use of the abstract form $\left.Q^{A}\right|_{\beta} ^{\alpha}$ is, indeed, more economical, this is a main reason why we suggest to apply it here. Only to show a final result after calculations the form (151) could be represented. Below we describe properties of the definition $\left.Q^{A}\right|_{\beta} ^{\alpha}$, which are necessary in the present paper.

The right hand side of the equation (151) shows that the quantities $\left.Q^{A}\right|_{\beta} ^{\alpha}$ are tensor densities of the same weight as the tensor densities $Q^{A}$. Therefore, the covariant derivative $\left(\left.Q^{A}\right|_{\beta} ^{\alpha}\right)_{; \gamma}$ of tensor densities $\left.Q^{A}\right|_{\beta} ^{\alpha}$ is defined in a usual manner as applied to a covariant quantity. Thus, the evident property

$$
\left.\left(Q_{; \alpha}^{A}\right)\right|_{\rho} ^{\tau}=\left(\left.Q^{A}\right|_{\rho} ^{\tau}\right)_{; \alpha}-\delta_{\alpha}^{\tau} Q_{; \rho}^{A}
$$


follows after covariant differentiation of (151). Also an action of a double vertical line is defined by a natural way:

$$
\left.\left.\left.\left.Q^{A}\right|_{\beta} ^{\alpha}\right|_{\nu} ^{\mu} \equiv\left(\left.Q^{A}\right|_{\beta} ^{\alpha}\right)\right|_{\nu} ^{\mu} \equiv Q^{B}\right|_{\nu} ^{\mu}
$$

where ' $B$ ' is a new generalized index. More important properties follow from the usual properties of the Lie derivative. Thus from

$$
\begin{aligned}
£_{\xi} \delta_{\tau}^{\rho} & =0 ; \\
£_{\xi}\left(Q^{A} P^{B}\right) & =P^{B} £_{\xi}\left(Q^{A}\right)+Q^{A} £_{\xi}\left(P^{B}\right) ; \\
£_{\zeta} £_{\xi}\left(Q^{A}\right)-£_{\xi} £_{\zeta}\left(Q^{A}\right) & =£_{[\zeta \xi]}\left(Q^{A}\right),
\end{aligned}
$$

where $[\zeta \xi]=\xi^{\rho} \zeta^{\alpha}, \rho-\zeta^{\rho} \xi^{\alpha}, \rho$, the next relations are derived:

$$
\begin{aligned}
\left.\delta_{\tau}^{\rho}\right|_{\beta} ^{\alpha} & =0 \\
\left.\left(Q^{A} P^{B}\right)\right|_{\beta} ^{\alpha} & =\left.\left(P^{B}\right)\right|_{\beta} ^{\alpha} Q^{A}+\left.\left(Q^{A}\right)\right|_{\beta} ^{\alpha} P^{B} ; \\
\left.\left.Q^{A}\right|_{\rho} ^{\beta}\right|_{\alpha} ^{\tau}-\left.\left.Q^{A}\right|_{\alpha} ^{\tau}\right|_{\rho} ^{\beta} & =\left.\delta_{\alpha}^{\beta} Q^{A}\right|_{\rho} ^{\tau}-\left.\delta_{\rho}^{\tau} Q^{A}\right|_{\alpha} ^{\beta}
\end{aligned}
$$

Among these the second property (158) is more useful. Thus, in our calculations frequently we use the transformations, like this

$$
\left.\frac{\partial \hat{\mathcal{L}}}{\partial Q_{B ; \alpha}} Q_{B}\right|_{\tau} ^{\rho}=\left.\left(\frac{\partial \hat{\mathcal{L}}}{\partial Q_{B ; \alpha}} Q_{B}\right)\right|_{\tau} ^{\rho}-\left.\left(\frac{\partial \hat{\mathcal{L}}}{\partial Q_{B ; \alpha}}\right)\right|_{\tau} ^{\rho} Q_{B} .
$$

The first term at the right hand side, to which the vertical line is applied, is a vector density, therefore it is useful also

$$
\left.\left(\frac{\partial \hat{\mathcal{L}}}{\partial Q_{B ; \alpha}} Q_{B}\right)\right|_{\tau} ^{\rho}=-\delta_{\tau}^{\rho} \frac{\partial \hat{\mathcal{L}}}{\partial Q_{B ; \alpha}} Q_{B}+\delta_{\tau}^{\alpha} \frac{\partial \hat{\mathcal{L}}}{\partial Q_{B ; \rho}} Q_{B} .
$$

The above properties are enough to derive the useful relation:

$$
\left.\frac{\partial\left(\left.Q^{A}\right|_{\beta} ^{\alpha}\right)}{\partial Q^{B}} Q^{B}\right|_{\tau} ^{\rho}=\left.\left.Q^{A}\right|_{\beta} ^{\alpha}\right|_{\tau} ^{\rho}
$$

The definition $\left.Q^{A}\right|_{\beta} ^{\alpha}$ in (150) corresponds to the presentation of the covariant derivative by the way:

$$
\bar{D}_{\alpha} Q^{A}=\partial_{\alpha} Q^{A}+\left.Q^{A}\right|_{\tau} ^{\rho} \bar{\Gamma}_{\alpha \rho}^{\tau}
$$

Namely this presentation is used to represent a partial derivative through a covariant one. Recall that for the vector density $\hat{Q}^{\alpha}: \bar{D}_{\alpha} \hat{Q}^{\alpha}=\partial_{\alpha} \hat{Q}^{\alpha}$, and for the antisymmetric tensor density $\hat{Q}^{\alpha \beta}: \bar{D}_{\beta} \hat{Q}^{\alpha \beta}=\partial_{\beta} \hat{Q}^{\alpha \beta}$. Then, the definition (163) evidently gives

$$
\begin{gathered}
\left.\hat{Q}^{\alpha}\right|_{\tau} ^{\rho} \bar{\Gamma}_{\alpha \rho}^{\tau}=0, \\
\left.\hat{Q}^{\alpha \beta}\right|_{\tau} ^{\rho} \bar{\Gamma}_{\beta \rho}^{\tau}=0 .
\end{gathered}
$$

Then (161), as an example of the vector density, gives:

$$
\left.\left(\frac{\partial \hat{\mathcal{L}}}{\partial Q_{B ; \alpha}} Q_{B}\right)\right|_{\tau} ^{\rho} \bar{\Gamma}_{\alpha \rho}^{\tau}=0
$$


At last, we note that the notation $\left.Q^{A}\right|_{\beta} ^{\alpha}$ can be used also in antisymmetrization of the covariant derivatives:

$$
Q_{; \mu \nu}^{A}-Q_{; \nu \mu}^{A}=\left.Q^{A}\right|_{\beta} ^{\alpha} \bar{R}_{\alpha}^{\beta}{ }_{\mu \nu}
$$

Calculations in the text of the paper are very prolonged and it is impossible to note in each the case what formulae in this Appendix have been used. Therefore, we do not do it, explaining only a general direction of calculations. The information in this Appendix is quite enough to repeat our calculations without principal obstacles.

\section{B Necessary formulae in the Einstein-Gauss-Bonnet gravity}

The action of the Einstein $D$-dimensional theory with a bare cosmological term $\Lambda_{0}$ corrected by the Gauss-Bonnet term (see, for example, [14]) is

$$
\begin{aligned}
& S=-\frac{1}{2 \kappa_{D}} \int d^{D} x \hat{L}_{E G B}+\int d^{D} x \hat{\mathcal{L}}_{m} \\
&=-\frac{1}{2 \kappa_{D}} \int d^{D} x \sqrt{-g}\left[R-2 \Lambda_{0}+\alpha(R R)_{G B}\right]+\int d^{D} x \hat{\mathcal{L}}_{m}, \\
& \quad(R R)_{G B} \equiv R_{\alpha \beta \gamma \delta} R^{\alpha \beta \gamma \delta}-4 R_{\alpha \beta} R^{\alpha \beta}+R^{2},
\end{aligned}
$$

where $\kappa_{D}=2 \Omega_{D-2} G_{D}>0$ and $\alpha>0 ; G_{D}$ is the $D$-dimension Newton's constant, $\Omega_{D-2}$ is the area of a unit $(D-2)$-dimensional sphere, and we restrict ourselves by $\Lambda_{0} \leq 0$. The subscript ' $E$ ' is related to the pure Einstein part of the action (168), and the subscript ' $G B$ ' is related to the Gauss-Bonnet part connected with $\alpha$-coefficient.

To present the metric Lagrangian $\hat{L}_{E G B}$ in an explicitly covariant form $\hat{\mathcal{L}}_{E G B}$ one has to change partial derivatives $\partial_{\mu}$ of the dynamic metric $g_{\mu \nu}$ in the Riemannian tensor by the covariant derivatives $\bar{D}_{\mu}$. We use next useful formulae:

$$
R_{\tau \rho \sigma}^{\lambda}=\bar{D}_{\rho} \Delta_{\tau \sigma}^{\lambda}-\bar{D}_{\sigma} \Delta_{\tau \rho}^{\lambda}+\Delta_{\rho \eta}^{\lambda} \Delta_{\tau \sigma}^{\eta}-\Delta_{\eta \sigma}^{\lambda} \Delta_{\tau \rho}^{\eta}+\bar{R}_{\tau \rho \sigma}^{\lambda}=\delta R_{\tau \rho \sigma}^{\lambda}+\bar{R}_{\tau \rho \sigma}^{\lambda}
$$

where

$$
\Delta_{\mu \nu}^{\alpha}=\Gamma_{\mu \nu}^{\alpha}-\bar{\Gamma}_{\mu \nu}^{\alpha}=\frac{1}{2} g^{\alpha \rho}\left(\bar{D}_{\mu} g_{\rho \nu}+\bar{D}_{\nu} g_{\rho \mu}-\bar{D}_{\rho} g_{\mu \nu}\right)
$$

is the difference between the Christoffel symbols related to the dynamic $g_{\mu \nu}$ and the background $\bar{g}_{\mu \nu}$ metrics. It is useful the next relations also. Analogously to (163) one defines the covariant derivative related to the dynamic metric:

$$
D_{\alpha} Q^{A}=\partial_{\alpha} Q^{A}+\left.Q^{A}\right|_{\tau} ^{\rho} \Gamma_{\alpha \rho}^{\tau} .
$$

Then, comparing (172) and (163) one obtains

$$
D_{\alpha} Q^{A}=\bar{D}_{\alpha} Q^{A}+\left.Q^{A}\right|_{\tau} ^{\rho} \Delta_{\alpha \rho}^{\tau}
$$

The coefficients $n^{*}$ and $m^{*}$ (see formulae (176) and (177) below), corresponding to the Lagrangian $\hat{\mathcal{L}}_{E G B}$ in (168), are necessary for calculating superpotentials. However, at the first it is useful to present the next derivatives:

$$
\begin{aligned}
& \frac{-2 \kappa_{D}}{\sqrt{-g}} \frac{\partial \hat{\mathcal{L}}_{E G B}}{\partial g_{\mu \nu ; \alpha}}=\frac{-2 \kappa_{D}}{\sqrt{-g}}\left(\frac{\partial \hat{\mathcal{L}}_{E}}{\partial g_{\mu \nu ; \alpha}}+\frac{\partial \hat{\mathcal{L}}_{G B}}{\partial g_{\mu \nu ; \alpha}}\right) \\
= & 2\left[\Delta_{\sigma \rho}^{\alpha} g^{\sigma[\rho} g^{\mu] \nu}+g^{\alpha \sigma} \Delta_{\sigma \rho}^{(\mu} g^{\nu) \rho}-g^{\alpha(\mu} \Delta_{\sigma \rho}^{\nu)} g^{\sigma \rho}\right] \\
+ & 4 \alpha\left[2 R^{\alpha \sigma \rho(\mu} \Delta_{\sigma \rho}^{\nu)}-\Delta_{\sigma \rho}^{\alpha} R^{\sigma \mu \nu \rho}\right]
\end{aligned}
$$




$$
\begin{aligned}
& -4 \alpha\left[2 R^{\alpha \sigma} \Delta_{\sigma \rho}^{(\mu} g^{\nu) \rho}-2 g^{\alpha(\mu} \Delta_{\sigma \rho}^{\nu)} R^{\sigma \rho}+2 g^{\alpha \sigma} \Delta_{\sigma \rho}^{(\mu} R^{\nu) \rho}-2 R^{\alpha(\mu} \Delta_{\sigma \rho}^{\nu)} g^{\sigma \rho}\right. \\
& \left.+\Delta_{\sigma \rho}^{\alpha} R^{\sigma \rho} g^{\mu \nu}+\Delta_{\sigma \rho}^{\alpha} g^{\sigma \rho} R^{\mu \nu}-2 \Delta_{\sigma \rho}^{\alpha} R^{\sigma(\mu} g^{\nu) \rho}\right] \\
& +4 \alpha R\left[\Delta_{\sigma \rho}^{\alpha} g^{\sigma[\rho} g^{\mu] \nu}+g^{\alpha \sigma} \Delta_{\sigma \rho}^{(\mu} g^{\nu) \rho}-g^{\alpha(\mu} \Delta_{\sigma \rho}^{\nu)} g^{\sigma \rho}\right] \\
& \frac{-2 \kappa_{D}}{\sqrt{-g}} \frac{\partial \hat{\mathcal{L}}_{E G B}}{\partial g_{\mu \nu ; \alpha \beta}}=\frac{-2 \kappa_{D}}{\sqrt{-g}}\left(\frac{\partial \hat{\mathcal{L}}_{E}}{\partial g_{\mu \nu ; \alpha \beta}}+\frac{\partial \hat{\mathcal{L}}_{G B}}{\partial g_{\mu \nu ; \alpha \beta}}\right) \\
= & {\left[g^{\alpha(\mu} g^{\nu) \beta}-g^{\alpha \beta} g^{\mu \nu}\right] } \\
+ & 2 \alpha\left[2 R^{\alpha(\mu \nu) \beta}-4 R^{\alpha(\mu} g^{\nu) \beta}+2 g^{\mu \nu} R^{\alpha \beta}+2 g^{\alpha \beta} R^{\mu \nu}+R\left(g^{\alpha(\mu} g^{\nu) \beta}-g^{\alpha \beta} g^{\mu \nu}\right)\right](1.5)
\end{aligned}
$$

Substituting (174) and (175) into (61) and (62) one obtains

$$
\begin{aligned}
\hat{n}_{\sigma}^{* \lambda \alpha \beta}= & (E)^{*} \hat{n}_{\sigma} \lambda \beta+(G B) \hat{n}_{\sigma}^{* \lambda \alpha \beta} \\
= & \left.\frac{\sqrt{-g}}{2 \kappa_{D}}\left\{g^{\alpha \beta} \delta_{\sigma}^{\lambda}-g^{\lambda(\alpha} \delta_{\sigma}^{\beta}\right)\right\} \\
+ & \frac{\alpha \sqrt{-g}}{\kappa_{D}}\left\{-2 R_{\sigma}{ }^{(\alpha \beta) \lambda}-4 R_{\sigma}^{\lambda} g^{\alpha \beta}+4 R_{\sigma}^{(\alpha} g^{\beta) \lambda}+R\left(g^{\alpha \beta} \delta_{\sigma}^{\lambda}-g^{\lambda(\alpha} \delta_{\sigma}^{\beta)}\right)\right\} \\
\hat{m}_{\sigma}^{* \alpha \beta}= & (E) \hat{m}_{\sigma}^{* \alpha \beta}+{ }_{(G B)} \hat{m}_{\sigma}^{* \alpha \beta} \\
= & -\frac{\sqrt{-g}}{2 \kappa_{D}}\left[\delta_{\sigma}^{\alpha} \Delta_{\rho \tau}^{\beta} g^{\rho \tau}-2 \Delta_{\sigma \rho}^{\alpha} g^{\beta \rho}+\Delta_{\rho \sigma}^{\rho} g^{\alpha \beta}\right] \\
& +\frac{2 \alpha \sqrt{-g}}{\kappa_{D}}\left[R^{\alpha \tau \rho}{ }_{\sigma} \Delta_{\tau \rho}^{\beta}-2 R^{\alpha(\tau \beta)}{ }_{\rho} \Delta_{\tau \sigma}^{\rho}\right] \\
& +\frac{4 \alpha \sqrt{-g}}{\kappa_{D}}\left[4 g^{\rho[\alpha} R_{\tau}^{\beta]} \Delta_{\rho \sigma}^{\tau}+2 R_{\sigma}^{[\alpha} g^{\tau] \rho} \Delta_{\tau \rho}^{\beta}+2 g^{\alpha[\beta} R_{\rho}^{\tau]} \Delta_{\tau \sigma}^{\rho}\right. \\
& \left.-g^{\tau \beta}\left(\bar{D}{ }_{(\tau} R_{\sigma)}^{\alpha}+R_{(\tau}^{\rho} \Delta_{\sigma) \rho}^{\alpha}-R_{\rho}^{\alpha} \Delta_{\tau \sigma}^{\rho}\right)\right] \\
& -\frac{\alpha \sqrt{-g}}{\kappa_{D}}\left[\left(\delta_{\sigma}^{\alpha} \Delta_{\rho \tau}^{\beta} g^{\rho \tau}-2 \Delta_{\sigma \rho}^{\alpha} g^{\beta \rho}+\Delta_{\rho \sigma}^{\rho} g^{\alpha \beta}\right) R-2 \delta_{\sigma}^{(\alpha} g^{\tau) \beta} \partial_{\tau} R\right]
\end{aligned}
$$

Using the coefficients (176) and (177) in (71) one obtains for a pure Nother canonical starred superpotential in EGB gravity

$$
\begin{aligned}
\hat{\imath}^{* \alpha \beta} & =\frac{1}{\kappa_{D}}\left(\hat{g}^{\rho[\alpha} \bar{D}_{\rho} \xi^{\beta]}+\hat{g}^{\rho[\alpha} \Delta_{\rho \sigma}^{\beta]} \xi^{\sigma}\right) \\
& -\frac{2 \alpha \sqrt{-g}}{\kappa_{D}}\left\{\Delta_{\lambda \sigma}^{\rho} R_{\rho}^{\lambda \alpha \beta}+4 \Delta_{\lambda \sigma}^{\rho} g^{\lambda[\alpha} R_{\rho}^{\beta]}+\Delta_{\rho \sigma}^{[\alpha} g^{\beta] \rho} R\right\} \xi^{\sigma} \\
& -\frac{2 \alpha \sqrt{-g}}{\kappa_{D}}\left\{R_{\sigma}^{\lambda \alpha \beta}+4 g^{\lambda[\alpha} R_{\sigma}^{\beta]}+\delta_{\sigma}^{[\alpha} g^{\beta] \lambda} R\right\} \bar{D}_{\lambda} \xi^{\sigma}, \\
\overline{\hat{\imath}^{* \alpha \beta}} & =\frac{1}{\kappa_{D}} \overline{D^{[\alpha} \hat{\xi}^{\beta]}}-\frac{2 \alpha}{\kappa_{D}}\left\{\overline{\hat{R}}_{\sigma}^{\lambda \alpha \beta}+\overline{4 g^{\lambda[\alpha} \hat{R}_{\sigma}^{\beta]}}+\delta_{\sigma}^{[\alpha} \overline{g^{\beta] \lambda} \hat{R}}\right\} \bar{D}_{\lambda} \xi^{\sigma} .
\end{aligned}
$$

To finalize forming a pure canonical superpotential one needs to choose the divergence. We prefer the choice induced by the Katz-Lifshits approach [19] instead of the choice in [17] (see discussions in $[20-22])$. Thus, we choose in (115) - (117)

$\hat{d}^{\alpha}={ }_{(E)} \hat{d}^{\alpha}+{ }_{(G B)} \hat{d}^{\alpha}=2 \Delta_{\tau \beta}^{[\tau} \hat{g}^{\alpha] \beta}+4 \alpha\left(\hat{R}_{\rho}^{\beta \tau \alpha}-2 \hat{R}_{\rho}^{[\tau} g^{\alpha] \beta}-2 \delta_{\rho}^{[\tau} \hat{R}^{\alpha] \beta}+\delta_{\rho}^{[\tau} g^{\alpha] \beta} \hat{R}\right) \Delta_{\tau \beta}^{\rho}$. 
Using the coefficients (176) and (177) in (95) one obtains the Belinfante corrected starred superpotential in EGB gravity

$$
\begin{aligned}
\hat{\imath}_{B}^{* \alpha \beta} & =\frac{1}{\kappa_{D}}\left[\left(\delta_{\sigma}^{[\alpha} \bar{D}_{\lambda} \hat{g}^{\beta] \lambda}-\bar{D}^{[\alpha} \hat{g}^{\beta] \rho} \bar{g}_{\rho \sigma}\right) \xi^{\sigma}+\hat{g}^{\lambda[\alpha} \bar{D}_{\lambda} \xi^{\beta]}\right] \\
& +\frac{\alpha}{\kappa_{D}} \bar{D}_{\lambda}\left\{\hat{R}_{\sigma}^{\lambda \alpha \beta}+4 g^{\lambda[\alpha} \hat{R}_{\sigma}^{\beta]}+\left[2 \hat{R}_{\tau}^{\rho \lambda[\alpha}-2 \hat{R}^{\rho \lambda}{ }^{[\alpha}-8 \hat{R}_{\tau}^{\lambda} g^{\rho[\alpha}\right.\right. \\
& \left.\left.+4 \hat{R}_{\tau}^{\rho} g^{\lambda[\alpha}+4 g^{\rho \lambda} \hat{R}_{\tau}^{[\alpha}+2 \hat{R}\left(\delta_{\tau}^{\lambda} g^{\rho[\alpha}-\delta_{\tau}^{\rho} g^{\lambda[\alpha}\right)\right] \bar{g}^{\beta] \tau} \bar{g}_{\rho \sigma}\right\} \xi^{\sigma} \\
& -\frac{2 \alpha}{\kappa_{D}}\left\{\hat{R}_{\sigma}^{\lambda \alpha \beta}+4 g^{\lambda[\alpha} \hat{R}_{\sigma}^{\beta]}+\delta_{\sigma}^{[\alpha} g^{\beta] \lambda} \hat{R}\right\} \bar{D}_{\lambda} \xi^{\sigma}, \\
\overline{\hat{\imath}_{B}^{* \alpha \beta}} & =\frac{1}{\kappa_{D}} \overline{\hat{g}^{\lambda[\alpha} D_{\lambda} \xi^{\beta]}}-\frac{2 \alpha}{\kappa_{D}}\left\{\overline{\hat{R}}_{\sigma}^{\lambda \alpha \beta}+4 \overline{g^{\lambda[\alpha} \hat{R}_{\sigma}^{\beta]}}+\delta_{\sigma}^{[\alpha} \overline{g^{\beta] \lambda} \hat{R}}\right\} \bar{D}_{\lambda} \xi^{\sigma} .
\end{aligned}
$$

\section{References}

1. A.N. Petrov, in Classical and Quantum Gravity, ed. by M.N. Christiansen, T.K. Rasmussen (Nova Science Publishers, New York, 2008), chap. 2, pp. 79-160. Preprint arXiv:0705.0019 [gr-qc]

2. L.B. Szabados, Living Rev. Relativity 12(4), 4 (2009). Online version: http://www.livingreviews.org/lrr-2009-4

3. J.B. Pitts, W.C. Schieve. Null cones in lorentz-covariant general relativity (2001). Preprint arXiv:gr-qc/0111004

4. P.G. Bergmann, Phys. Rev. 112(1), 287 (1958)

5. A. Komar, Phys. Rev. 113(3), 934 (1959)

6. A. Trautman, in Gravitation: an Introduction to Current Research, ed. by L. Witten (John Wiley and Sons, New York - London, 1962), chap. 5, pp. 169-198

7. S. Deser, Gen. Relat. Grav. 1(1), 9 (1970). Preprint gr-qc/0411023

8. L.P. Grishchuk, A.N. Petrov, A.D. Popova, Commun. Math. Phys. 94(3), 379 (1984)

9. J. Katz, J. Bičák, D. Lynden-Bell, Phys. Rev. D 55(10), 5957 (1997). Preprint arXiv:grqc/0504041

10. C.M. Chen, J.M. Nester, Class. Quantum Grav. 16(4), 1279 (1999). Preprint arXiv:grqc/9809020

11. C.M. Chen, J.M. Nester, Grav. Cosmol. 6(4), 257 (2000). Preprint arXiv:gr-qc/0001088

12. L. Fatibene, M. Ferraris, M. Francaviglia, M. Raiteri, J. Math. Phys. 42(3), 1173 (23pp) (2001). Preprint arXiv:gr-qc/0003019

13. A.N. Petrov, J. Katz, Proc. R. Soc. A, London 458(2018), 319 (2002). Preprint arXiv:grqc/9911025

14. S. Deser, B. Tekin, Phys. Rev. D 67(8), 084009 (7pp) (2003). Preprint arXiv:hepth/0212292

15. D. Lovelock, J. Math. Phys. 12(3), 498 (4pp) (1971)

16. T.P. Sotiriou, V. Faraoni, Rev. Mod. Phys. 82(1), 451 (2010)

17. N. Deruelle, J. Katz, S. Ogushi, Class. Quantum Grav. 21(8), 1971 (2004). Preprint arXiv:gr-qc/0310098

18. L. Fatibene, M. Ferraris, M. Francaviglia, Int. J. Geom. Meth. Mod. Phys. 2(3), 373 (2005). Preprint arXiv:math-ph/0411029

19. J. Katz, G.I. Livshits, Class. Quantum Grav. 25(17), 175024 (17pp) (2008). Preprint arXiv:0807.3079 [gr-qc]

20. A.N. Petrov, Class. Quantum Grav. 26(7), 135010 (16pp) (2009). Corrigendum in: [21]; Preprint arXiv:0905.3622 [gr-qc]

21. A.N. Petrov, Class. Quantum Grav. 27(6), 069801 (2pp) (2010). Corrigendum to the ref. [20]

22. A.N. Petrov, Grav. Cosmol. 16(1), 34 (2010). Preprint arXiv:0911.5419 [gr-qc]

23. A.N. Petrov, Class. Quantum Grav. 28(7), 215021 (17pp) (2011). Preprint arXiv:1102.5636 [gr-qc] 
24. N.V. Mitzkevich, Physical Fields in General Theory of Relativity (Nauka, Moscow, 1969). In Russian

25. J.R. Ray, Nuovo Cim. A 56(1), 189 (1968)

26. F.J. Belinfante, Physica 6(9), 887 (1939)

27. D.G. Boulware, S. Deser, Phys. Rev. Lett. 55(24), 2656 (1985)

28. F. Klein, Nachr. d. Konig. Gesellsch. d. Wiss. zu Gottingen, Math-phys. Klasse pp. 171-189 (1918). Reprinted in: [29], pages 568-585

29. F. Klein, Gesammelte Mathematische Abhandlungen. Band 1 (Springer, Berlin, 1921)

30. Y. Kosmann-Schwarzbach, The Noether Theorems. Sources and studies in the history of mathematics and physics (Springer, New York - Dordrecht - Heidelberg - London, 2011). Translated by Bertram E. Schwarzbach

31. N.P. Konopleva, V.N. Popov, Gauge Fields (Atomizdat, Moscow, 1980). In Russian

32. N. Byers. E. noether's discovery of the deep connection between symmetries and conservation laws (1998). Preprint arXiv:physics/9807044 [physics.hist-ph]; (21pp)

33. K. Brading, H.R. Brown. Noether's theorems and gauge symmetries (2000). Preprint arXiv:hep-th/0009058; (16pp)

34. K. Brading, H.R. Brown, in Symmetries in Physics. Philosophical reflections, ed. by K. Brading, E. Castellani (CUP, Cambridge, 2003), pp. 89-109

35. G. Sardanashvily, Int. J. Geom. Methods Mod. Phys. 6(6), 1047 (2009). Preprint arXiv:0906.1732 [math-ph]

36. E. Noether, Nachr. d. Konig. Gesellsch. d. Wiss. zu Gottingen, Math-phys. Klasse pp. 235-257 (1918). In German. English translation in: [30], pages 3-19

37. P. Von Freud, Ann. of Math. 40(2), 417 (1939)

38. A. Papapetrou, Proc. R. Irish Acad. A 52(2), 11 (1948)

39. A. Paddila, Class. Quantum Grav. 20(14), 3129 (2003). Preprint arXiv:gr-qc/0303082

40. G. Allemandi, M. Francaviglia, M. Raiteri, Class. Quantum Grav. 20(23), 5103 (2003). Preprint arXiv:gr-qc/0308019

41. N. Okuyama, J.I. Koga, Phys. Rev. D 71(8), 084009 (9pp) (2005). Preprint arXiv:hepth/0501044 